\title{
Uncertainty in processing relative clauses across East Asian languages
}

\author{
Jiwon Yun • Zhong Chen • Tim Hunter • \\ John Whitman • John Hale
}

Received: 3 October 2012 / Accepted: 4 October 2013 / Published online: 23 December 2014 (C) The Author(s) 2015. This article is published with open access at Springerlink.com

\begin{abstract}
The processing difficulty profile for relative clauses in Chinese, Japanese and Korean represents a challenge for theories of human parsing. We address this challenge using a grammar-based complexity metric, one that reflects a minimalist analysis of relative clauses for all three languages as well as structure-dependent corpus distributions. Together, these define a comprehender's degree of uncertainty at each point in a sentence. We use this idea to quantify the intuition that people do comprehension work as they incrementally resolve ambiguity, word by word. We find that downward changes to this quantitative measure of uncertainty derive observed processing contrasts between Subject- and Object-extracted relative clauses. This demonstrates that the complexity metric, in conjunction with a minimalist grammar and corpus-based weights, accounts for the widely-observed Subject Advantage.
\end{abstract}

Electronic supplementary material The online version of this article (doi:10.1007/s10831-014-9126-6) contains supplementary material, which is available to authorized users.

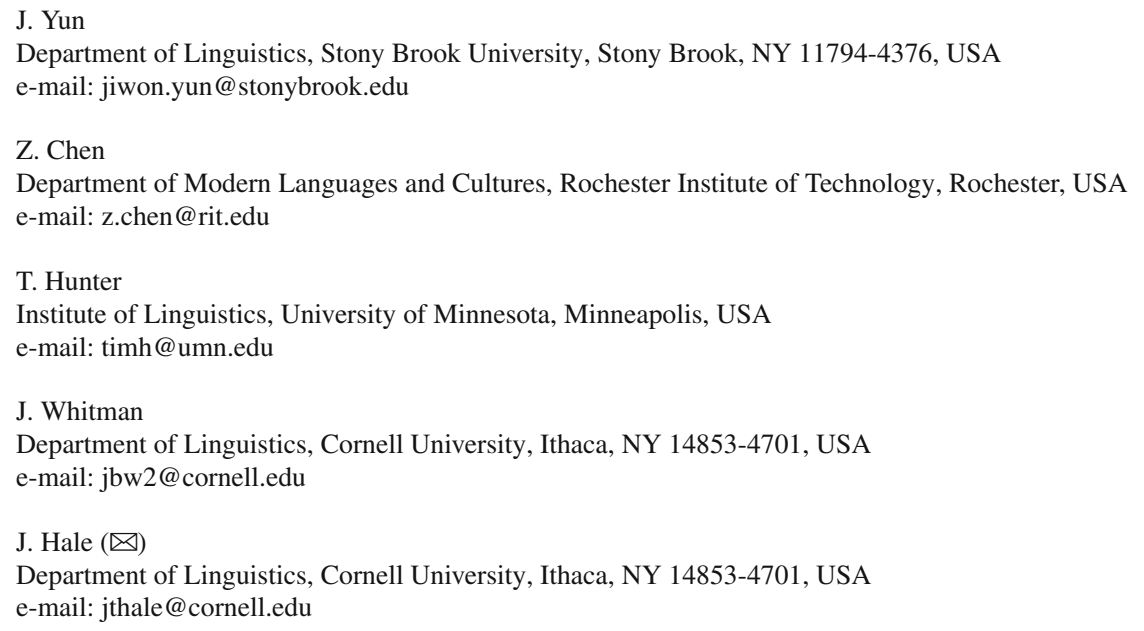


Keywords Relative clause $\cdot$ Information theory $\cdot$ Minimalism $\cdot$ Chinese $\cdot$ Japanese $\cdot$ Korean

\section{Introduction}

Relative clauses present linguists with a variety of puzzles. Two in particular are fundamental in the sense that any solution to them would carry implications for syntax, typology and psycholinguistics. The first is their structure. Can an analysis be given that is simultaneously general, consistent and descriptively accurate across multiple languages? The second is their processing. Why are some relative clauses easier to understand than others? Can asymmetries in processing be related to their syntactic structure and, if so, how? This paper proposes a solution to these puzzles. The basic idea is that subject and object relatives have different amounts of sentence-medial ambiguity. We show that a quantitative version of this idea can account for the observed processing asymmetry between these two sentence types.

To show this, we use Minimalist Grammars (Stabler 1997) to explicitly define the set of alternative syntactic analyses that are consistent with the sentence-initial substring of words perceived so far. Probability distributions over this remainder set characterize the expectations that a comprehender would have, partway through the sentence. To estimate them, we weight the grammars' structural rules in accordance with corpora like the Penn Chinese Treebank 7 (Xue et al. 2005), the Kyoto Corpus 4 (Kurohashi and Nagao 2003) and the Penn Korean Treebank 2 (Han et al. 2002). The uncertainty or entropy of this remainder set quantifies the notion of sentence-medial ambiguity. If this entropy goes down in the transition from one word to the next, then sentence-processing work must have occurred (Wilson and Carroll 1954; Hale 2006).

These assumptions suffice to derive a universal Subject Advantage in processing. Keenan and Hawkins (1974/1987) found early support for such a universal, and since then a growing body of experimental work has supported it. Proceeding from these findings, this paper fleshes out a role for minimalist syntax in a quantitative theory of derivational uncertainty. In particular, the proposed theory uses syntactic alternatives that are logically entailed by the grammar to derive numerical predictions of processing difficulty at specific words.

These predictions, across Chinese, Japanese \& Korean (henceforth: CJK) are laid out in Section 6. But, before that, Section 2 reviews the relevant psycholinguistics literature. Section 3 goes on to discuss the syntactic analysis of relative clauses involving subject and object gaps. For such constructions, there is a fairly strong consensus in the generative literature that some kind of extraction is implicated in the syntactic derivation (see Huang et al. 2009 for Chinese, Whitman 2012 for Japanese and Han and Kim 2004 for Korean). Section 4 introduces relative frequency estimation, a simple way of using corpora to put weights on grammar rules. This weighting makes it possible to quantify sentence-medial ambiguity as the entropy of the remainder set. Section 5 reviews the Entropy Reduction hypothesis, which links fluctuating entropy levels and processing difficulty. Finally, Section 7 concludes with a reflection on human sentence processing as information processing. Our grammars and corpus data are included as Electronic Supplementary Material, and our software is freely available. 


\section{Asymmetries in processing relative clauses}

\subsection{Relative clauses and the subject advantage}

In a relative clause (RC), a noun phrase is said to have been "relativized" from one of a number of different "underlying" positions, for example subject position or object position. The RC construction as a whole thus exhibits a Filler-Gap relationship. A large literature documents the finding that subject relatives (SRCs) are consistently easier to process than object relatives (ORCs) across languages, a processing asymmetry often referred to as the Subject Advantage. This Subject Advantage has been observed for English using a variety of different measures, including: reading times (King and Just 1991), eye-tracking (Traxler et al. 2002), ERP (King and Kutas 1995), fMRI (Just et al. 1996) and PET (Stromswold et al. 1996). European languages other than English also attest the Subject Advantage, for instance Dutch (Frazier 1987; Mak et al. 2002), French (Frauenfelder et al. 1980) and German (Schriefers et al. 1995; Mecklinger et al. 1995).

In all these languages, the RC appears after the noun it modifies. That is to say: English, French, German and Dutch all have postnominal RCs. By contrast, CJK RCs come before the noun they modify. This prenominal positioning of the $\mathrm{RC}$ with respect to the head noun is illustrated below ${ }^{1}$ in Examples 1-3.

(1) Chinese

a. SRC

[ $e_{i}$ shushi furen de] (jingli $i_{i}$ )

knows tycoon DE manager/someone

'the manager/someone who knows the tycoon'

b. ORC

[furen shushi $e_{i}$ de] (jingli ${ }_{i}$ )

tycoon knows DE manager/someone

'the manager/someone who the tycoon knows'

(2) Japanese

a. SRC

[ $e_{i}$ daigisi o hinansita] kisya $i$ senator Acc criticize reporter

'the reporter who criticized the senator'

\footnotetext{
1 Parentheses in Example 1 indicate the optionality of the head noun in Chinese. The other abbreviations in the glosses are:

Nom nominative case marker

Acc accusative case marker

Decl declarative verb ending

Adn adnominal verb ending
}

See also Table 4. 
b. ORC

[daigisi ga $e_{i}$ hinansita] kisya $i$

senator Nom criticize reporter

'the reporter who the senator criticized'

(3) Korean

a. SRC

[ $e_{i}$ uywon ul pinanhan] kica $_{i}$ senator Acc criticize.Adn reporter

'the reporter who criticized the senator'

b. ORC

[uywon i $e_{i}$ pinanhan] kica $_{i}$

senator Nom criticize.Adn reporter

'the reporter who the senator criticized'

The distinction between prenominal and postnominal positioning makes CJK RCs a uniquely valuable domain for testing universalist claims about human sentence processing. Below, we summarize some key empirical findings. Section 2.3 then goes on to assess the available theories.

\subsection{The subject advantage in CJK}

A variety of experiments have measured the Subject Advantage at specific points in sentences containing prenominal relative clauses. For instance, using self-paced reading, Ishizuka et al. (2003) and Miyamoto and Nakamura (2003, 2013) found it at the head noun in Japanese RCs. Ishizuka (2005) reports it as well at the RC-initial case-marked noun phrase. Korean is much the same: Kwon et al. (2006), for instance, report a Subject Advantage at the head noun. These contrasts have been replicated across several different methodologies including ERP in Japanese (Ueno and Garnsey 2008), and eye-tracking in Korean (Kwon et al. 2010).

The processing of Chinese RCs, on the other hand, has been harder to pin down. Early work by F. Hsiao and Gibson (2003) reported the inverse result, an Object Advantage, in contrast to the Subject Advantage found in Lin and Bever (2006). Further analysis indicates that this outcome may have been due to uncontrolled factors, such as local ambiguities (C. Lin and Bever 2011; Qiao et al. 2012; Vasishth et al. 2013; Y. Hsiao et al. 2014) $)^{2}$ and syntactic priming from the context with different thematic orders (Lin 2014). With stimuli that control local ambiguities such as the availability of argument omission, Jäger et al. (in press) observe a robust Subject Advantage in Chinese RCs. A wider array of references is provided below in Table 1. Overall, the weight of the evidence seems to suggest that Chinese is not exceptional after all but, rather, confirms the universalist view: the Subject Advantage manifests itself in both prenominal and postnominal RCs.

\footnotetext{
2 These sorts of ambiguities represent an exciting research area for CJK psycholinguistics. For a review with special emphasis on Japanese, see Hirose (2009).
} 
Table 1 Psycholinguistic experiments on Chinese relative clauses

\begin{tabular}{ll}
\hline Subject advantage & Object advantage \\
\hline C. Lin and Bever (2006; 2007; 2011) & F. Hsiao and Gibson (2003) \\
C. Lin (2008; submitted) & B. Chen et al. (2008) \\
F. Wu (2009) & Y. Lin and Garnsey (2011) \\
F. Wu et al. (2012) & Packard et al. (2011) \\
Vasishth et al. (2013) & Qiao et al. (2012) \\
Jäger et al. (in press) & Gibson and H. Wu (2013) \\
F. Wu and Kaiser (submitted) & \\
\hline
\end{tabular}

Table 2 Processing principles proposed for relative clauses

\begin{tabular}{|c|c|c|}
\hline \multicolumn{2}{|r|}{ Broad Categories } & General Proposals \\
\hline WORD ORDER & $\begin{array}{l}\text { Bever (1970); } \\
\text { MacDonald and Christiansen (2002) }\end{array}$ & $\begin{array}{l}\text { The sequence of words in SRCs is } \\
\text { closer to the canonical word order } \\
\text { than that in ORCs. }\end{array}$ \\
\hline $\begin{array}{l}\text { PARALLEL } \\
\text { FunCTION }\end{array}$ & Sheldon (1974) & $\begin{array}{l}\text { SRCs are easier to process than } \\
\text { ORCs because their head nouns play } \\
\text { the same role in both the main clause } \\
\text { and the subordinate clauses. }\end{array}$ \\
\hline $\begin{array}{l}\text { Perspective } \\
\text { Maintenance }\end{array}$ & MacWhinney $(1977,1982)$ & $\begin{array}{l}\text { SRC structures maintain the human } \\
\text { perspective and should be easier to } \\
\text { process than those that shift it, e.g. } \\
\text { ORCs. }\end{array}$ \\
\hline $\begin{array}{l}\text { ACCESSIBILITY } \\
\text { HIERARCHY }\end{array}$ & Keenan and Comrie (1977) & $\begin{array}{l}\text { Universal markedness hierarchy of } \\
\text { grammatical relations ranks the rel- } \\
\text { ativization from subject higher. }\end{array}$ \\
\hline \multirow[t]{2}{*}{$\begin{array}{l}\text { MEMORY } \\
\text { BURDEN }\end{array}$} & $\begin{array}{l}\text { LINEAR DisTANCE: } \\
\text { Wanner and Maratsos (1978); } \\
\text { Gibson (2000); } \\
\text { Lewis and Vasishth (2005) }\end{array}$ & \multirow[t]{2}{*}{$\begin{array}{l}\text { ORCs are harder because they impose } \\
\text { a greater memory burden. }\end{array}$} \\
\hline & $\begin{array}{l}\text { STRUCTURAL DistancE: } \\
\text { O'Grady (1997); Hawkins (2004) }\end{array}$ & \\
\hline \multirow{3}{*}{$\begin{array}{l}\text { STRUCTURAL } \\
\text { FREQUENCY }\end{array}$} & $\begin{array}{l}\text { Tuning Hypothesis: } \\
\text { Mitchell, Cuetos, Corley, and Brys- } \\
\text { baert (1995); Jurafsky (1996) }\end{array}$ & $\begin{array}{l}\text { SRCs occur more frequently than } \\
\text { ORCs and therefore are more ex- } \\
\text { pected and easier to process. }\end{array}$ \\
\hline & $\begin{array}{l}\text { SURPRISAL: } \\
\text { Hale (2001); Levy (2008) }\end{array}$ & $\begin{array}{l}\text { ORCs are more difficult because they } \\
\text { require a low-probability rule. }\end{array}$ \\
\hline & $\begin{array}{l}\text { ENTROPY REDUCTION: } \\
\text { Hale (2006) }\end{array}$ & $\begin{array}{l}\text { ORCs are harder because they force } \\
\text { the comprehender through more con- } \\
\text { fusing intermediate states. }\end{array}$ \\
\hline
\end{tabular}

\subsection{Theories of relative clause processing}

A variety of general principles have been advanced to account for the Subject Advantage. Table 2 catalogs some of the leading ideas. Among these, recent work has been especially concerned with MEMORY BURDEN and STRUCTURAL FREQUENCY. The memory burden idea, while appealing for European languages, does not work for CJK RCs that are prenominal. To see this, consider the distance between the head noun and its coindexed empty category $e_{i}$ in any of the Examples $1-3$. In all cases, the pre- 
dicted memory burden in the SRC would be greater than in the ORC, contrary to the observed empirical pattern.

The structural frequency idea is also appealing, since by and large the attestation rate of SRCs exceeds ORCs. However, in its most well-known incarnation as "surprisal" this idea also fails to derive the observed data. Levy (2008) acknowledges the situation when he writes

One way of interpreting these mixed results is to hypothesize that surprisal has a major effect on word-by-word processing difficulty, but that truly non-local (i.e., long-distance) syntactic dependencies such as relativization and WH-question formation are handled fundamentally differently $[\ldots]$

page 1166

At least in Levy's formulation, surprisal does not work for English RCs. Seeking a more adequate complexity metric, Hale (2003, 2006) advances an alternative called Entropy Reduction. Like surprisal, Entropy Reduction is information-theoretical. But unlike surprisal it correctly derives the observed Subject Advantage in English. Section 2.4 reviews the original account given on pages 116-118 of Hale (2003) in light of subsequent work.

\subsection{Entropy reduction as a complexity metric}

The basic idea of Entropy Reduction is that comprehenders struggle against the tide of ambiguity that they face in the course of incremental processing. Words, as they come in, are either helpful or unhelpful in narrowing down the interpretation that the speaker (or writer) intends. As in Hale (2006), we consider intermediate stages that correspond to sentence-initial substrings. Example 4 illustrates these stages with an example used in the account of English RC processing difficulty from that paper.

(4) initial substrings of "the sailor who s ship Jim take -ed have -ed one leg"
a. (empty string)
b. the
c. the sailor
d. the sailor who
e. the sailor who $\mathrm{s}$

The symbols in 4 mostly correspond to whole words, although some morphemes such as the genitive $s$ get their own symbol. From this perspective, the latest symbol added to the initial substring is taken to be 'informative' about the overall structure of the unfolding sentence. This contribution is quantified by changes in the conditional entropy of the derivation given the initial string.

The basic idea has a long history. To the best of our knowledge, it was introduced in Section 5.3 of Wilson and Carroll (1954). At this time, many cognitive scientists were 
interested in applying information theory to human communication (see for example Chap. 5 of Cherry (1961), Section 6.1 of Levelt (1974, volume II), or Chap. 3 of Smith (1973)). Wilson and Carroll applied Entropy Reduction to an artificial language of their own creation, by way of introducing the idea and demonstrating its potential utility for morphosyntactic analysis. In doing so, they also acknowledged a major restriction: their formulation relied on a Markov model of language, analogous to beads-on-astring.

Hale (2003) revived the Entropy Reduction idea by lifting this restriction and applied it to the analysis of processing asymmetries in English, including the Subject Advantage. This paper used context-free phrase structure grammars as models of language structure. The account of the Subject Advantage essentially turned on the possibility of recursive modification which exists in the SRC but which is eliminated by the embedded verb in the ORC. Later papers such as Hale (2006) upgraded the language model yet further to expressive formalisms like Stabler's Minimalist Grammars (1997) where a movement analysis of relativization can be stated directly. This change was accompanied by new algorithms for computing the metric, but the basic equation between human sentence processing work and the reduction of derivational uncertainty remained the same.

Section 9.1 of Hale (2006) notes that Entropy Reduction can derive the repetition accuracy cline that is observed along Keenan and Comrie's (1977) well-known Accessibility Hierarchy of relativizable grammatical relations $(\mathrm{AH})$. This same pattern is also part of the empirical support for the minimize domains (MiD) principle (Hawkins, 2004, Section 7.2). Entropy Reduction and MiD both derive the AH but from different starting points. Whereas MiD (and its predecessor, Early Immediate Constituents) considers the number of syntactic nodes involved in the ultimately-correct analysis of e.g. an English RC, Entropy Reduction takes into account changing distributions on intermediate parser states. It quantifies the idea of sentence-medial ambiguity with a numerical uncertainty level over intermediate parser states.

There is no necessary connection between Entropy Reduction as a complexity metric and Minimalist Grammars as a formalism. In fact, Frank (2013) recently applied Entropy Reduction to the analysis of British readers' eye fixation times using Simple Recurrent Nets as substitute for a grammar. In this study, Entropy Reduction emerged as a significant predictor of fixation duration. Interestingly, as he computed Entropy Reductions with greater and greater fidelity, Frank found that their fit to observed fixation times got better and better. This sort of result strengthens our confidence in the metric itself.

What remains to be shown is that Entropy Reduction derives the correct predictions in the key domain of prenominal RCs. This is important because, as things stand, there exists no formalized account of incremental processing difficulty that accords with data on both prenominal and postnominal RC processing. In order to make these predictions, we first have to fix upon a syntactic analysis. The syntactic analysis, discussed in Section 3, motivates a particular series of corpus studies (Section 4) aimed at weighting the rules of a formalized grammar fragment. 


\section{Structure of relative clauses}

As noted in the Introduction, there is a general consensus in the generative literature that movement is involved in the derivation of at least some CJK RCs. This evidence comes from the existence of island effects as well as subtler effects such as scope reconstruction and idiom chunk effects (Huang et al., 2009; Whitman, 2012; Han and Kim, 2004). Notice, however, that the consensus claim is not exhaustive. Other RCs seem to present island violations. A widespread view holds that these are cases of resumptive pro, where movement is blocked. But we restrict ourselves here to Subject and Object gaps in non-island environments. In these cases a movement analysis is appropriate.

\subsection{The promotion analysis}

There are two main movement analyses of RCs in the generative literature. An analysis moving a null operator from the position of the gap into the clausal projection of the RC was popular through the 1990s (Ning (1993) for Chinese, Ishii (1991) for Japanese, Kaplan and Whitman (1995) for Japanese and Korean). Kayne (1994) revives a different movement analysis for RCs, dating back to Brame (1967), Schachter (1973) and Vergnaud (1974). Under this "promotion" analysis, the RC head is moved directly from the position of the gap into RC head position. Kayne points out that this analysis is particularly attractive for prenominal RCs because it explains the well-known typological fact that prenominal relatives never include overt relative pronouns at the beginning of the RC. According to Kayne's analysis, this is because the RC head is first moved from TP to the specifier of CP. TP is then fronted around the head. The derivation thereby accounts for the head/RC order, the absence of relative pronouns at the beginning of the $\mathrm{RC}$, and the absence of evidence that prenominal RCs are CPs, rather than simple TPs. A further, theory-internal advantage of the promotion analysis is that it avoids a violation of the Extension Condition (Chomsky 1993) inherent in at least some operator movement analyses. Under such analyses, the RC is adjoined to the head of the nominal projection (or in some analyses, generated in Spec, DP). Relative operator movement then takes place within the CP, but this movement is not at the edge (the structurally highest position) inside the nominal projection. On the promotion analysis, by contrast, the NP generated in the position of the gap moves consistently to the edge of the projection at each step of the derivation.

Promotion analyses have been adopted by X. Wu (2000) for Chinese, and Hoshi (1995) for Japanese. Huang et al. (2009) adopt a mixed analysis of Chinese RCs, involving both promotion and operator adjunction. As our purpose in this paper is to generalize across comparable derivations, in the formal grammar fragments, we implemented promotion derivations, as representative of the currently most widelyadopted analysis of RCs in minimalist theory. Figure 1 sketches these derivations with Korean and Japanese presented at the same time in 1(a) and Chinese separately in 1(b). 


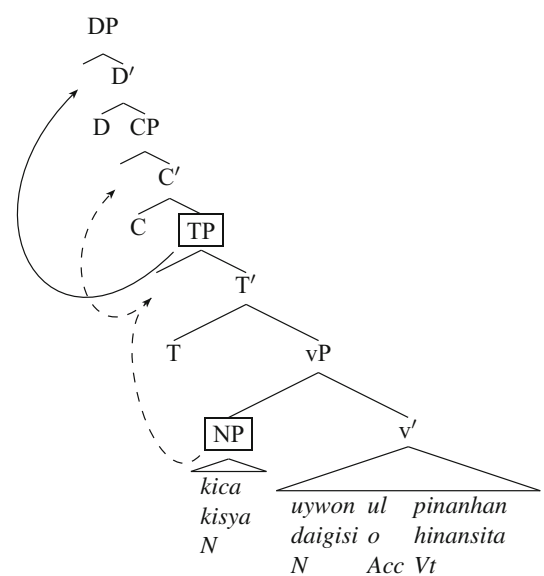

(a) Korean and Japanese

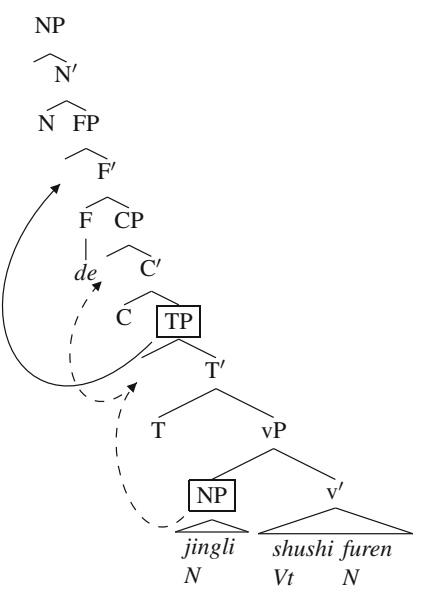

(b) Chinese

Fig. 1 Syntactic analyses of CJK relative clauses

\subsection{Minimalist grammars}

The promotion analysis discussed above in Section 3.1 is a compelling idea about the general structure of RCs across languages. But in order to extract specific quantitative predictions, one must express this general idea in some concrete way. In this project, we did this using the Minimalist Grammars (MGs) of Stabler (1997). This system formalizes certain themes of Chomsky's Minimalist Program (1995). For instance, MG lexical entries have sequences of features that motivate derivational operations. One kind of feature motivates the MERGE operation; this aspect is reminiscent of categorial grammar (Berwick and Epstein, 1995). Another kind of feature motivates the MOVE operation; this operation reorganizes the derived tree and has no direct parallel in categorial grammar. In our grammars, for instance, there is a +wh feature that motivates movement of a corresponding - wh phrase. The finitude of the available feature types, in any given grammar, is crucial for reining in the expressive power of these grammars.

Minimalist Grammars can be viewed as defining two operations (MERGE, MOVE) in a kind of bottom-up way. But this perspective is not exclusive. An important mathematical result shows that the same formalism can also be viewed as a rewriting system that works top-down (Michaelis 2001; Harkema 2001). Neither direction is privileged if one adopts a view of MG derivations as trees. One can get a sense of these derivation trees by imagining nodes labeled with the operation name (MERGE or MOVE) and leaves labeled with feature bundles. ${ }^{3}$

The derivation tree viewpoint underwrites the interpretation of MGs as stochastic branching processes, and therefore also their interpretation as probabilistic

\footnotetext{
${ }^{3}$ See Fig. 12 of Hale (2006). The distinction between derivation tree and derived tree was introduced into cognitive science by Aravind Joshi.
} 
grammars. ${ }^{4}$ The core idea is that a derivation may continue, from the top down, by application of any one of a number of alternative operations. These operations are "backwards" from the usual bottom-up perspective but this presents no difficulty. If appropriately normalized, the weights on these alternative branches become probabilities and the grammars themselves can take on a more cognitive interpretation: they define the class of structures a comprehender might be expecting. ${ }^{5}$ In order to set up this cognitive interpretation (Section 5), numerical weights have to be determined. Section 4 explains how we used corpus distributions to obtain these numbers.

\section{From corpora to weighted grammars}

The step from corpora to weighted grammars follows a simple logic: branches of the derivation tree represent choices about which structure to generate. In a performance model, these choices might reasonably be based on experience. We can approximate this experience by estimating various statistics from Treebanks or other samples. This section illustrates one such procedure, starting from a simplified example based on context-free grammars. It turns out, however, this is not really a simplification at all, because the weighting of MG derivations proceeds in exactly the same manner.

\subsection{Relative frequency estimation for tree branches}

Because the fragments of Chinese, Japanese and Korean are expressed as MGs, their derivations may be viewed as having been generated by a context-free phrase structure grammar $(\mathrm{CFG})$. The estimation problem therefore reduces to the problem of weighting a CFG. But this problem is easy to solve; the method can be demonstrated with a small example such as Fig. 2. The rules in Fig. 2 present us with choices: is an NP going to have a determiner (Det) or will it be a bare noun (N)? Similarly, will a verb phrase be transitive or intransitive? The idea of relative frequency estimation is to set the weights on these choices according to the ratio of those two structure types in a sample. This task is far easier if one has access to a Treebank - a corpus whose sentences are annotated with phrase structures.

Given a Treebank, one can easily weight this grammar by counting. First, count how many times an NP node appears with two daughters, one labeled Det and one labeled $\mathrm{N}$; say this number is 100 . Second, count how many times an NP node appears with a single $\mathrm{N}$ daughter; say this number is 50. Similarly, suppose that a VP node appears

\footnotetext{
4 Chapter 2 of Harris (1963) treats, in detail, the sort of branching process used in these linguistic models. Smith (1973, pages 66-68) and Hale (2006, Section 3.1) both review the work of Grenander (1967) who was the first to see the connection to branching processes.

5 The modeling in this paper relies exclusively on syntactic information e.g. grammatical category, hierarchical phrase structure and WH-movement. This leaves out nonsyntactic factors, such as animacy and information structure, which also play a crucial role in human sentence comprehension. However, nothing in the overall approach prevents inclusion of additional features in the formal grammar fragment e.g. diacritics such as ANIMATE or TOPIC. In continuing modeling work, Z. Chen (2014) estimates weighted grammars with formalist as well as "functionalist" feature names. The results accurately reflect the Subject Advantage and the animacy effect in English, Italian and Chinese RCs.
} 


$\begin{aligned} \mathrm{S} & \rightarrow \mathrm{NP} \mathrm{VP} \\ \mathrm{NP} & \rightarrow \mathrm{Det} \mathrm{N} \\ \mathrm{NP} & \rightarrow \mathrm{N} \\ \mathrm{VP} & \rightarrow \mathrm{V} \\ \mathrm{VP} & \rightarrow \mathrm{V} \mathrm{NP}\end{aligned}$

Fig. 2 Unweighted grammar offers alternative ways to rewrite both NP and VP

with a single V daughter 120 times, and that a VP node appears with a V daughter and an NP daughter 90 times. These counts can be summarized as shown in (5).

\begin{tabular}{|l|r|}
\hline NP with two daughters, labeled Det and N & 100 \\
NP with a single daughter, labeled N & 50 \\
\hline VP with a single daughter, labeled V & 120 \\
VP with two daughters, labeled V and NP & 90 \\
\hline
\end{tabular}

Then we would assign the 'NP $\rightarrow$ Det N' rule a weight of $\frac{100}{150}$ (or $\frac{2}{3}$ ), and the 'NP $\rightarrow$ N' rule a weight of $\frac{50}{150}$ ( or $\frac{1}{3}$ ). Similarly, the two VP rules would be assigned weights of $\frac{120}{210}$ and $\frac{90}{210}$ respectively. Note that when a nonterminal, such as $\mathrm{S}$ in this example, has only one possible expansion, then this rule must have a weight of one and so there is no need to consult a corpus.

The situation is analogous for the more complex MG rewriting system: counts of how often certain grammatical patterns appear in corpora still suffice to determine the relevant weights. To illustrate, the relevant corpus frequencies for our Korean MG are given in (6). For simplicity, we omit from (6) the details of the rewriting system's parent-daughter combinations that are indicated explicitly in Fig. 2 and (5), and instead describe the relevant grammatical patterns in formalism-neutral terms (e.g. "intransitive verb", "complement clause"). The key point is that these counts determine the weights for our Korean grammar in precisely the same way that the structural counts in (5) determined the weights for the grammar in Fig. 2. As in Fig. 2 the counts are grouped into alternatives; each decision in the stochastic derivation process requires choosing from one of these pairs. Setting weights for each of the two alternatives at each such choice point fully determines a probability distribution over the derivations of our grammars, among which are the RC derivations discussed in Section 3.

(6)

\begin{tabular}{|l|r|}
\hline intransitive verb & 1417 \\
transitive verb & 1038 \\
\hline pro in subject position & 594 \\
non-pro noun phrase in subject position & 961 \\
\hline pro in object position & 23 \\
non-pro noun phrase in object position & 1015 \\
\hline subject relative clause & 1030 \\
object relative clause & 130 \\
\hline relative clause & 1160 \\
complement clause & 902 \\
\hline noun phrase with complement clause or relative clause & 2062 \\
noun phrase consisting of only a simple noun & 1976 \\
\hline
\end{tabular}


Although MGs differ from CFGs (e.g. by involving movement operations) counts of how often certain grammatical patterns appear in corpora still suffice to determine the weights of the relevant grammatical decisions. For example, note that one of the decisions that appears in our Korean grammar is the choice between SRCs and ORCs. While this can be thought of as choosing a gap position, in an MG this decision amounts to the choice between two different node labels on the same derivation tree node. In this more complex case, we again compute weights simply by counting how many SRCs and how many ORCs appear in a corpus and normalizing appropriately.

\subsection{Counting constructions in corpora}

To see how this counting is accomplished, this section offers two examples. These examples are from Korean, although the procedure for the Japanese and Chinese grammars is very similar. The chief consideration to keep in mind is the distinction between the formalism in which the Treebank is encoded (typically phrase structure) and the formalism being applied in the linguistic performance model (here, MGs).

The first example is transitivity. How can we estimate the rate at which VPs are transitive as opposed to intransitive? Looking in the Penn Korean Treebank (KTB) (Han et al., 2002) we identify particular structural configurations that the annotators used to flag these alternatives. ${ }^{6}$ These configurations are schematically illustrated below in $7 \mathrm{a}$ and $7 \mathrm{~b}$.

(7) a. transitive configuration

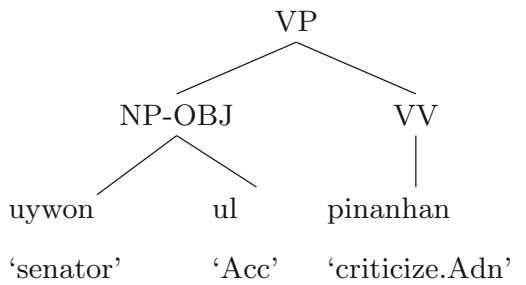

b. intransitive configuration

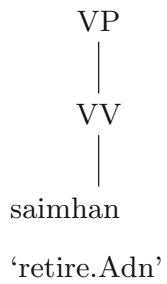

The count of configuration $7 \mathrm{a}\left(C_{a}\right)$ compared to configuration $7 \mathrm{~b}\left(C_{b}\right)$ estimates the transitivity parameter that we would need as a weight in our grammar. In a grammar like the one in Fig. 2 we would assign $\frac{C_{a}}{C_{a}+C_{b}}$ to the transitive VP rule 'VP $\rightarrow$ V NP' and $\frac{C_{b}}{C_{a}+C_{b}}$ to other, intransitive rule ' $\mathrm{VP} \rightarrow \mathrm{V}$ '.

\footnotetext{
6 We employ the pattern matching tool Tregex (Levy and Andrew, 2006) for our corpus search.
} 
The second example is relativization from Subject as opposed to Object. Here the procedure is exactly the same. The KTB represents SRCs as an S node which (i) adjoins to an NP, and (ii) has as its first daughter a WH-operator that is coindexed with a trace in an NP-SBJ position; similarly for ORCs and an NP-OBJ position. Specific cases of these two patterns are shown below in $8 \mathrm{a}$ and $8 \mathrm{~b}$. Of course, the criterial features are the tree configurations and the coindexation-not the specific words. These structures are the ones that qualify as SRCs or ORCs in the sense required for the totals shown in (6).

(8) a. SRC configuration

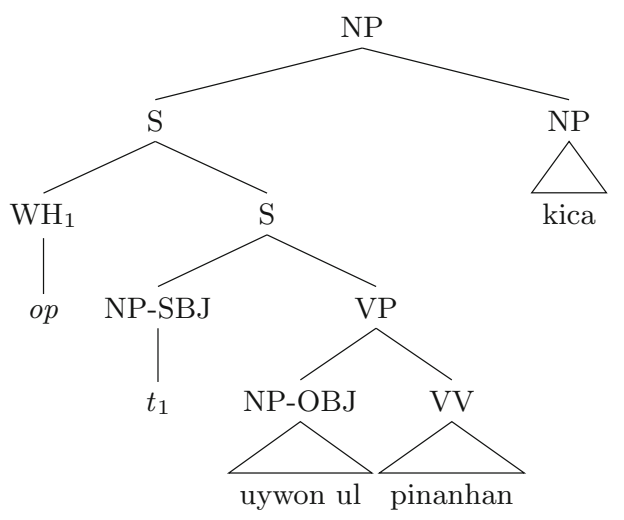

b. ORC configuration

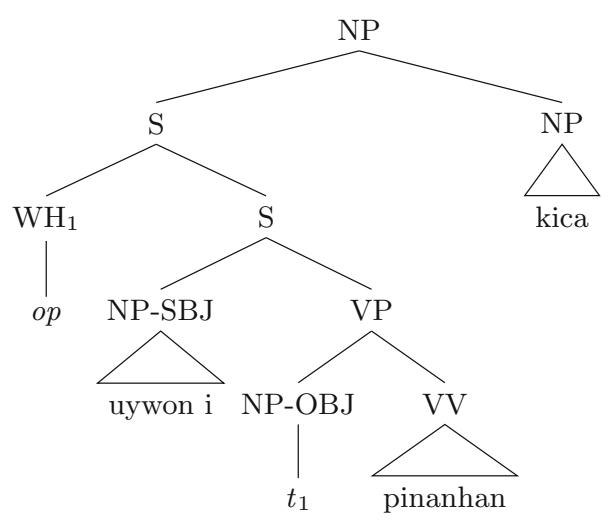

Appendix 1 includes more details e.g. about weighting the Japanese and Chinese grammars. The logic of the approach is identical, but we have restricted attention to Korean here because certain technical details make it the simplest of the three cases.

\section{Weighted grammars and information gain}

The Entropy Reduction hypothesis, referred to above in Section 2.4, requires some sort of weighting to quantify degrees of expectation. Section 4 introduced the weight- 
setting methodology. But equally required for a solid understanding of Entropy Reduction is the idea of conditioning grammars on initial substrings of sentences. This aspect, taken up in this section, is symbolized by the vertical dimension in Table 3.

Entropy Reduction is an incremental complexity metric; this means that it makes predictions on a word-by-word basis. These predictions reflect a quantity-entropycalculated over a set of derivations that remain "in play" at each successive word in a sentence. If no words have been heard, then the set of in-play derivations is, of course, the full set of all possible derivations. But as the comprehender considers successive words of an initial substring, these words begin to impose more of a constraint. With an explicit grammar fragment, we can calculate the remainder set of allowable word-sequences that can grammatically follow some given initial substring. We call members of this set "remainders." The remainders are the strings that would be generated by a new grammar representing the intersection of the given initial string with the original, full-sentence grammar (Bar-Hillel et al., 1964). This intersecting, in the style of Bar-Hillel, is indicated in the transition from the upper-left quadrant to the lower-left quadrant of Table 3. Each remainder has at least one derivation associated with it, and these derivations are compactly encoded by what is called an intersection grammar. In this paper, the yield condition is that the first few words must match an explicitly-given list representing the words that have already been heard or read, as in example 4. We call intersection grammars meeting this condition "remainder grammars." Transitioning to the bottom-right quadrant of Table 3, we add weights. This change does not alter the requirement that each derivation in the remainder grammar should remain consistent with the same initial string. But importantly, the weights can now quantify the degree to which an idealized comprehender would expect one remainder or another.

The information gained from a word in a sentence is precisely the drop in derivational uncertainty that is precipitated by that word. This uncertainty is formalized using the definition of entropy $(H)$ given below in 9 .

$$
H(X)=-\sum_{i} p\left(x_{i}\right) \log _{2} p\left(x_{i}\right)
$$

Table 3 Generative grammar and two augmentations of it

\begin{tabular}{|c|c|}
\hline $\begin{array}{c}\frac{\text { (Generative) Grammar }}{\text { discrete formal system }} \\
\text { generates a sentence if a derivation } \\
\text { exists }\end{array}$ & $\begin{array}{l}\text { discrete formal system with weights } \\
\text { generates (sentence,weight) pairs } \\
\text { sentence-weights accumulate } \\
\text { rule-weights }\end{array}$ \\
\hline $\begin{array}{l}\frac{\text { Intersection Grammar }}{\text { generates a sentence if a derivation }} \\
\text { exists } \\
\text { subject to a condition on its yield }\end{array}$ & $\begin{array}{l}\text { Weighted Intersection Grammar } \\
\text { generates a (sentence,weight) pair } \\
\text { if a derivation exists } \\
\text { and the yield-condition is satisfied }\end{array}$ \\
\hline
\end{tabular}




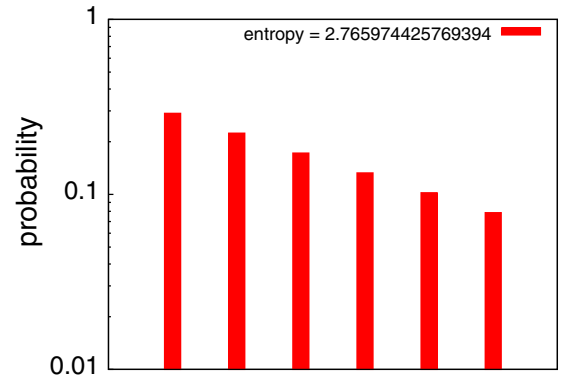

(a) Entropy $=2.8 \mathrm{bits}$

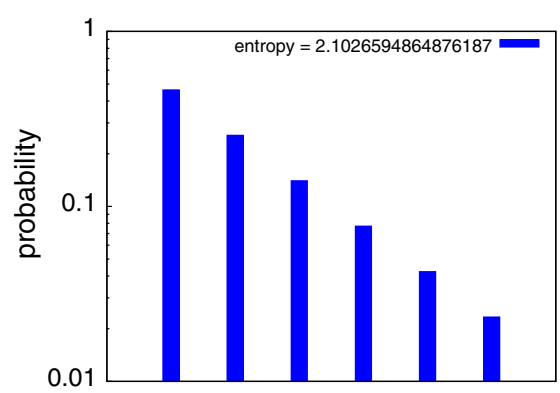

(b) Entropy $=2.1 \mathrm{bits}$

Fig. 3 Two distributions, one of which is more peaked

In this application to performance models of language, the $p\left(x_{i}\right)$ in definition 9 are the probabilities of syntactic derivations that are still "in play" at a given initial substring. Using the methods of Grenander (1967) and Nederhof and Satta (2008), it causes no particular difficulty if the number of these derivations is unbounded (see Appendix 2).

Weighted grammars thus define a probability distribution on what might be coming up later in the sentence, assuming it turns out to be grammatical. If we graph this probability distribution in a way such that each remainder-derivation $x_{i}$ has its own bar and the height of the bars correspond to their probability $p\left(x_{i}\right)$ then we can interpret the entropy of this distribution visually as the flatness of the graph. Given two distributions over the same events, the distribution with the flatter graph is the higher-entropy distribution. Figure 3 illustrates this sort of comparison with two distributions with geometrically-decreasing probabilities, just like those defined by the weighted grammars for CJK. By visual inspection, one can see how the lower entropy distribution 3(a) concentrates its probability on the left-hand side of the graph more heavily than the higher entropy distribution 3(b) does, the latter being more spread out. The maximum entropy distribution is of course the uniform distribution which, if graphed, would be a flat horizontal line.

If a particular word ends up tightening the overall constraints on the remainder set, then entropy has been reduced. Ultimately, if the sentence is unambiguous then uncertainty about the intended derivation should be brought down to zero by the end of the utterance. But locally, words that open up more options than they close down can cause entropy to increase. In some interpretations of information theory this is interpreted as "negative information"; however, as its name implies, Entropy Reduction only considers transitions on which information is actually gained. Appendix 3 goes into additional aspects of information theory.

\section{Processing predictions}

This section reports the incremental processing difficulty profiles that derive from the syntactic analysis discussed in Section 3 via the Entropy Reduction complexity metric reviewed in Section 2.4. This general methodology is discussed at length in Hale (2006). In all three languages the pattern is the same: a Subject Advantage is derived 
Table 4 Abbreviations

\begin{tabular}{|c|c|c|c|}
\hline \multicolumn{2}{|c|}{ Descriptive labels for terminal nodes } & \multicolumn{2}{|c|}{ Symbols appearing in derived strings } \\
\hline $\mathrm{N}$ & noun & $t$ & trace; indicates movement's launching site \\
\hline V & verb & pro & unpronounced pronoun; not derived by movement \\
\hline & -t: transitive $\quad-\mathrm{i}$ : intransitive & $e$ & empty category, unspecified \\
\hline & -d: declarative $\quad-n$ : adnominal & {$[\quad]$} & brackets indicate embedding \\
\hline fact & $\begin{array}{l}\text { nouns such as fact that take a } \\
\text { complement clause }\end{array}$ & \multicolumn{2}{|c|}{ Syntactic Constructions } \\
\hline de & relativizer in Chinese & $\mathrm{SRC}$ & Subject Relative Clause \\
\hline Nom & nominative case marker & ORC & Object Relative Clause \\
\hline Acc & accusative case marker & NCC & Noun Complement Clause \\
\hline Dem & demonstratives & \multicolumn{2}{|c|}{ Linking Hypothesis } \\
\hline $\mathrm{Cl}$ & classifiers & ER & Entropy Reduction \\
\hline Time & temporal phrases & & \\
\hline
\end{tabular}

such that SRCs are correctly identified as easier to understand. The following subsections discuss in detail the positions at which these Subject Advantages are predicted - typically at the beginning of the relative clause region and at its head noun. The discussion relates these predicted incremental processing asymmetries to grammatical alternatives defined by a formal grammar fragment. We focus on the role of sentence structure in processing by using grammar fragments that derive part-of-speech symbols, rather than particular lexical items. A complete list of these symbols is provided in Table 4.

\subsection{Korean}

The word-by-word difficulty predictions for Korean RCs using Entropy Reduction are shown in Fig. 4. This figure illustrates that more processing effort is predicted for ORCs than SRCs in general. Specifically, a Subject Advantage stands out on the second word (i.e. case marker) and the fourth word (i.e. head noun). The rest of this section details the derivation of these processing asymmetries at these specific positions.

Figure 5 tracks how the most probable structures and their probabilities change as the comprehender encounters new words. Each box lists sentence-level strings generated by the remainder grammar at the given initial substring, highlighted in bold, along with the entropy of the probability distribution over those remainders. These entropy values assist in the interpretation of the entropy reductions (ER) shown on each arrow, which are the actual difficulty predictions graphed in Fig. 4. The strings also include non-boldface words that are anticipated but as yet unheard. Each alternative is ranked by the conditional probability of the whole structure given the common initial substring. For instance, when readers have only encountered the noun symbol $N$ as the first word $\left(w_{1}\right)$, the most likely full sentence is a simple intransitive clause, N Nom Vid (probability 0.563). 

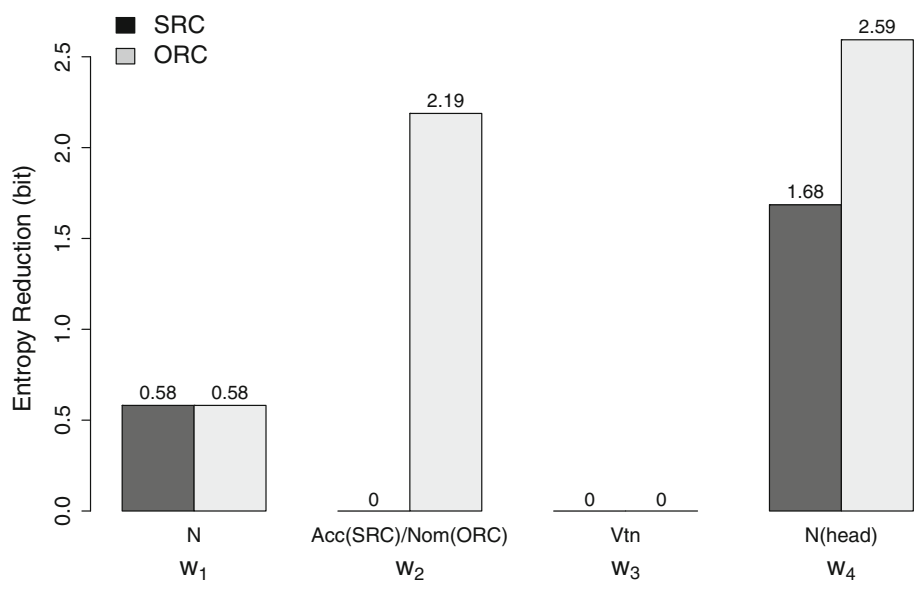

Fig. 4 ER prediction for Korean RCs

The Subject Advantage at the case marker $\left(w_{2}\right)$ can be explained as follows. More processing difficulty is predicted for ORCs at $w_{2}$ because remainder-set entropy is reduced in the transition from $w_{1}$ to $w_{2}$ when processing the ORC (ER 2.18 bits), whereas there is no reduction in the case of the SRC. Since both SRC and ORC start with the same word at $w_{1}$ (i.e. $N$ ), the prediction rests on the difference in the entropy value at $w_{2}$. In other words, the Subject Advantage at $w_{2}$ is due to the lower entropy of the ORC-initial substring N Nom (4.210 bits), compared to the SRC-initial substring $N$ Acc (11.073 bits). This difference in entropy can be understood by reference to remainder sets at $w_{2}$, as shown in Fig. 6. This figure illustrates that while N Nom is very likely to be a matrix subject $(0.713)$, the remainder distribution at $N$ Acc is less concentrated. Therefore, the ORC-initial substring at $w_{2}$ is associated with a lower entropy value than the corresponding SRC-initial substring, which leads to more Entropy Reduction in the ORC's transition from $w_{1}$ to $w_{2}$.

The Subject Advantage at the head noun $\left(w_{4}\right)$ is also indicated by greater Entropy Reduction for the ORC in the transition from $w_{3}$ to $w_{4}$. In this transition, the remainder-set entropy is reduced more for the ORC (ER 2.59 bits) than for the SRC (ER 1.68 bits). At $w_{3}$, the entropy is higher for the ORC (14.827 bits) than for the SRC (12.905 bits). Once the head noun is revealed at $w_{4}$, the entropy is still higher for the ORC but the difference in entropy between the two states becomes relatively small (11.220 bits for SRC and 12.234 bits for ORC). Thus, the predicted Subject Advantage at the head noun derives mainly from entropy differences at the verb immediately preceding it.

This explanation of the Subject Advantage at the head noun accords with that given in Yun et al. (2010) (YWH). YWH attributed the ORC processing penalty to higher entropy due to additional possible remainders at the verb. ${ }^{7} \mathrm{YWH}$ observed that in the

\footnotetext{
7 These "additional" remainders are members of the set-difference between two sets that, due to recursion, are infinite. As Appendix 2 discusses in further detail, this engenders neither philosophical nor practical difficulty.
} 


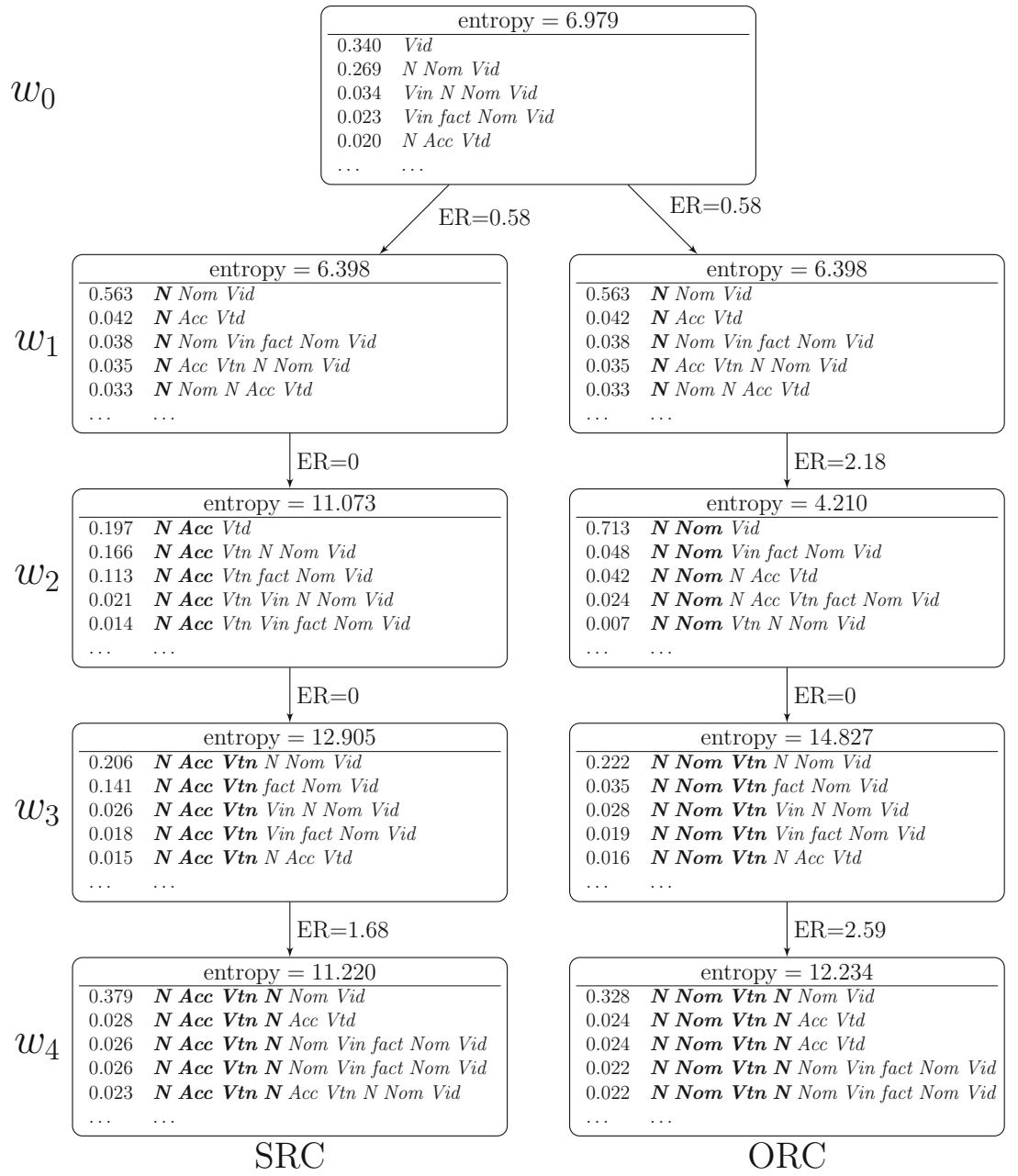

Fig. 5 Likely derivations and their conditional probabilities in Korean RCs

SRC prefix NAcc Vtn the case-marked noun is an argument of the embedded transitive verb as in (9), whereas in the ORC-initial substring N Nom Vtn there is an additional possibility that the case-marked noun is in fact a matrix subject, where both the subject and the object of the embedded clause are omitted, as in (10). Since verbal arguments may be freely omitted in Korean when they are recoverable from the context, it is not unreasonable to suppose that this additional possibility, with multiple null elements, indeed plays a role. YWH contains examples of sentences that correspond to the additional structure in (10b) as in (11a), (11b), and (11c). Indeed, adnominal clauses with null elements both in subject and object positions are attested in the corpus as shown in Appendix 1. 


\begin{tabular}{|lll|}
\hline Probability & Remainder & Type \\
\hline 0.197 & pro $\boldsymbol{N} \boldsymbol{A c c}$ Vtd & simplex SOV with Sbj-pro \\
0.166 & {$[t \boldsymbol{N} \boldsymbol{A c c}$ Vtn $]$ N Nom Vid } & SRC \\
0.113 & {$[$ pro $\boldsymbol{N} \boldsymbol{A c c}$ Vtn $]$ fact Nom Vid } & NCC with Sbj-pro \\
0.021 & {$[t \boldsymbol{N} \boldsymbol{A c c}$ Vtn $][t$ Vin $]$ N Nom Vid } & stacked SRCs \\
0.014 & {$[t \boldsymbol{N} \boldsymbol{A c c}$ Vtn $][$ pro Vin $]$ fact Nom $N$ Acc Vtd } & SRC / NCC with Sbj-pro \\
$\ldots$ & $\ldots$ & $\ldots$ \\
\hline entropy $=11.073$ & \\
\hline
\end{tabular}

(a) SRC-initial substring $N A c c$

\begin{tabular}{|lll|}
\hline Probability & Remainder & Type \\
\hline 0.713 & N nom Vid & simplex SV \\
0.048 & {$[$ nom Vin $]$ fact Nom Vid } & NCC \\
0.042 & $\boldsymbol{N}$ nom $N$ Acc Vtd & simplex SOV \\
0.024 & {$[$ N nom $N$ Acc Vtn $]$ fact Nom Vid } & NCC \\
0.007 & {$[\boldsymbol{N}$ nom $t$ Vtn $]$ N Nom Vid } & ORC \\
$\ldots$ & $\ldots$ & $\ldots$ \\
\hline entropy $=4.210$ & \\
\hline
\end{tabular}

(b) ORC-initial substring $N$ Nom

Fig. 6 Possible remainders at the second word for Korean RCs

(9) SRC-initial substring N Acc Vtn
a. [e N Acc Vtn]

(10) ORC-initial substring N Nom Vtn
a. [N Nom $e$ Vtn]
b. N Nom [e e Vtn]

(11) Additional possible structures for the ORC-initial substring

a. SRC with Sbj-pro

kica ka [e e kongkyekhan] uywon ul manassta. reporter Nom $t$ pro attack.Adn senator Acc meet.Decl

'The reporter met the senator who attacked someone.'

b. ORC with Obj-pro

kica ka [e e kongkyekhan] uywon ul manassta. reporter Nom pro $t$ attack.Adn senator Acc meet.Decl

'The reporter met the senator whom someone attacked.'

c. Noun Complement Clause (NCC) with Sbj- and Obj-pro

kica ka [e e kongkyekhan] sasil ul alkoissta. reporter Nom pro pro attack.Adn fact Acc know.Decl

'The reporter knows the fact that someone attacked someone.'

The present study confirms this account by tracking all remainders, at each initial substring, as shown in Fig. 7. This figure illustrates that the ORC-initial substring licenses more remainders than does the corresponding SRC-initial substring at the same level of embedding. The remainders ranked 6, 17, and 21 in the (b) panel of 


\begin{tabular}{|llll|}
\hline Rank & Probability & Remainder & Type \\
\hline$\ldots$ & $\ldots$ & $\ldots$ \\
5 & 0.015 & pro $[t \boldsymbol{N}$ Acc $\boldsymbol{V t n}]$ N Acc Vtd & SRC \\
$\ldots$ & $\ldots$ & $\ldots$ & $\ldots$ \\
11 & 0.010 & pro $[$ pro $\boldsymbol{N} \boldsymbol{A c c} \boldsymbol{V t n}]$ fact Acc Vtd & NCC with Sbj-pro \\
$\ldots$ & $\ldots$ & $\ldots$ & $\ldots$ \\
\hline entropy & $=12.905$ & & \\
\hline
\end{tabular}

(a) SRC-initial substring $N$ Acc Vtn

\begin{tabular}{|llll|}
\hline Rank & Probability & Remainder & Type \\
\hline$\ldots$ & $\ldots$ & $\ldots$ & $\ldots$ \\
5 & 0.016 & pro $[$ N Nom $t$ Vtn $]$ N Acc Vtd & ORC \\
6 & 0.016 & N Nom $[$ pro $t$ Vtn $]$ N Acc Vtd & ORC with Sbj-pro \\
$\ldots$ & $\ldots$ & $\ldots$ & $\ldots$ \\
17 & 0.004 & N Nom $[$ t pro Vtn $]$ N Acc Vtd & SRC with Obj-pro \\
$\ldots$ & $\ldots$ & $\ldots$ & $\ldots$ \\
20 & 0.003 & pro $[$ N Nom pro Vtn $]$ fact Acc Vtd & NCC with Obj-pro \\
21 & 0.003 & N Nom $[$ pro pro Vtn $]$ fact Acc Vtd & NCC with Sbj- and Obj-pro \\
$\ldots$ & $\ldots$ & $\ldots$ & $\ldots$ \\
\hline entropy $=14.827$ & & \\
\hline
\end{tabular}

(b) ORC-initial substring $N$ Nom Vtn

Fig. 7 Selected possible remainders at the third word for Korean RCs

Fig. 7 correspond to the additional structures (11a)-(11c) that were originally identified in Yun et al. (2010). Part of the ambiguity due to these additional structures is resolved at the next word as the possibility of an NCC is eliminated when the head noun $N$ is heard. This contributes to greater Entropy Reduction for the ORC at the head noun.

\subsection{Japanese}

Entropy Reduction also predicts the Subject Advantage in Japanese. The word-byword processing difficulty predictions for Japanese RCs are shown in Fig. 8. The pattern here is very similar to the Korean case discussed in Section 6.1: the Subject Advantage is predicted to show up at the same structural positions as in the Korean examples, namely at the second word (i.e. case marker) and at the fourth word (i.e. head noun). Figure 9 tracks how the most probable structures and their probabilities change as the comprehender encounters new words in Japanese.

The Subject Advantage at the case marker $\left(w_{2}\right)$ is predicted because remainder-set entropy is reduced in the transition from $w_{1}$ to $w_{2}$ when processing the ORC (ER 1.26 bits), whereas there is no reduction in the case of the SRC. As in Korean, greater processing difficulty for the ORC at $w_{2}$ is attributable to the lower entropy of the ORC-initial substring $N$ Nom (4.430 bits), compared to the SRC-initial substring $N$ Acc (5.773 bits) since the entropy at $w_{1}$ is the same for the SRC and the ORC (5.688 bits). Although the difference in entropy at $w_{2}$ is not immediately obvious from the very top-ranked parses as it was in the case of Korean, the source of the difference turns out to be similar with the Korean case if we continue by examining some lower- 

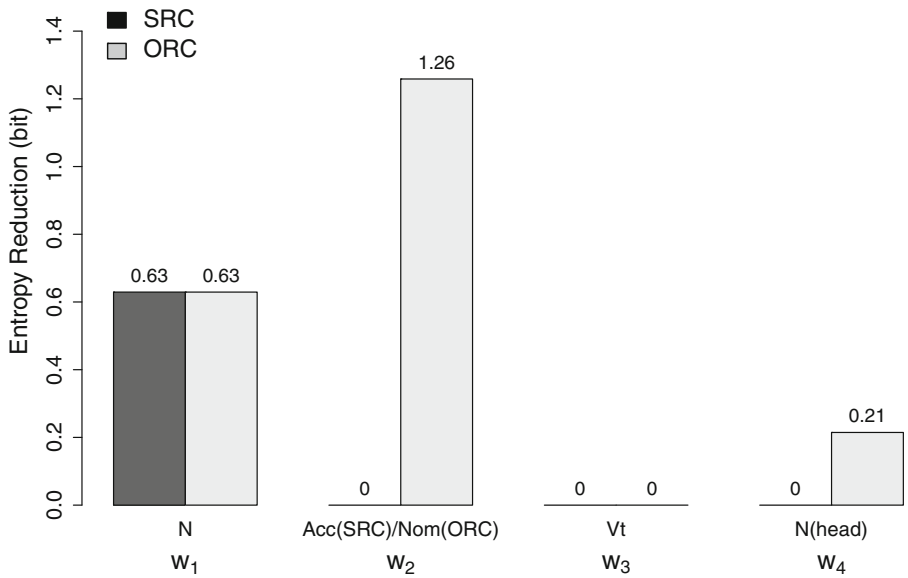

Fig. 8 ER prediction for Japanese RCs

order derivations. Figure 10 shows all analyses with a probability over 0.001 for both SRC and ORC initial substrings at the case marker. The smaller cardinality (31 vs 42) of nontrivial derivations in the ORC already suggests that more work has been done, resulting in a more highly-organized parser state. Further inspection of Fig. 10 shows that the remainders of N Nom tend to concentrate their probability mass on interpretations of that string as a subject of a simple sentence. On the other hand, the remainders of $N A c c$ exhibit a broader spread over more complex derivations involving more levels of embedding. The difference between these probability distributions creates the Subject Advantage at the case marker, in much the same way as in Korean. Korean and Japanese seem to differ here only in degree.

The sources for the Subject Advantage at the head noun $\left(w_{4}\right)$ are, however, not exactly parallel with Korean, due to a distinctive characteristic of Japanese. Note that in Japanese, adnominal and declarative forms are the same for verbs. Thus when the third word (i.e. the verb) is heard, this sentence-initial substring is ambiguous between adnominal clauses (i.e. relative clause or noun complement clauses) and declarative clauses. Figure 11 shows that both the SRC and ORC at $w_{3}$ are most likely to be interpreted as declarative sentences at this point. However, the distribution of the remainder set is more concentrated around a simple declarative analysis in the case of the SRC-initial substring (0.544), compared to the ORC-initial substring (0.256). This difference in the distribution of the remainder sets reflects the asymmetrical distribution of subject and object pros in Japanese: subject pro is abundant, while object pro is less so. Appendix 1 details how this distributional difference manifests itself in Japanese corpora. If a comprehender detects a missing subject in the sentence, the empty category is likely to be pro, whereas a missing direct object is rather ambiguous between an RC gap and pro. Thus, a hearer can be more certain about the rest of the sentence having heard the SRC-initial substring $N A c c V t$, which lacks an overt subject, compared to the ORC-initial substring N Nom Vt, which lacks an overt direct object. The lower entropy at $w_{3}$ for the SRC-initial substring (5.773 bits) than for 


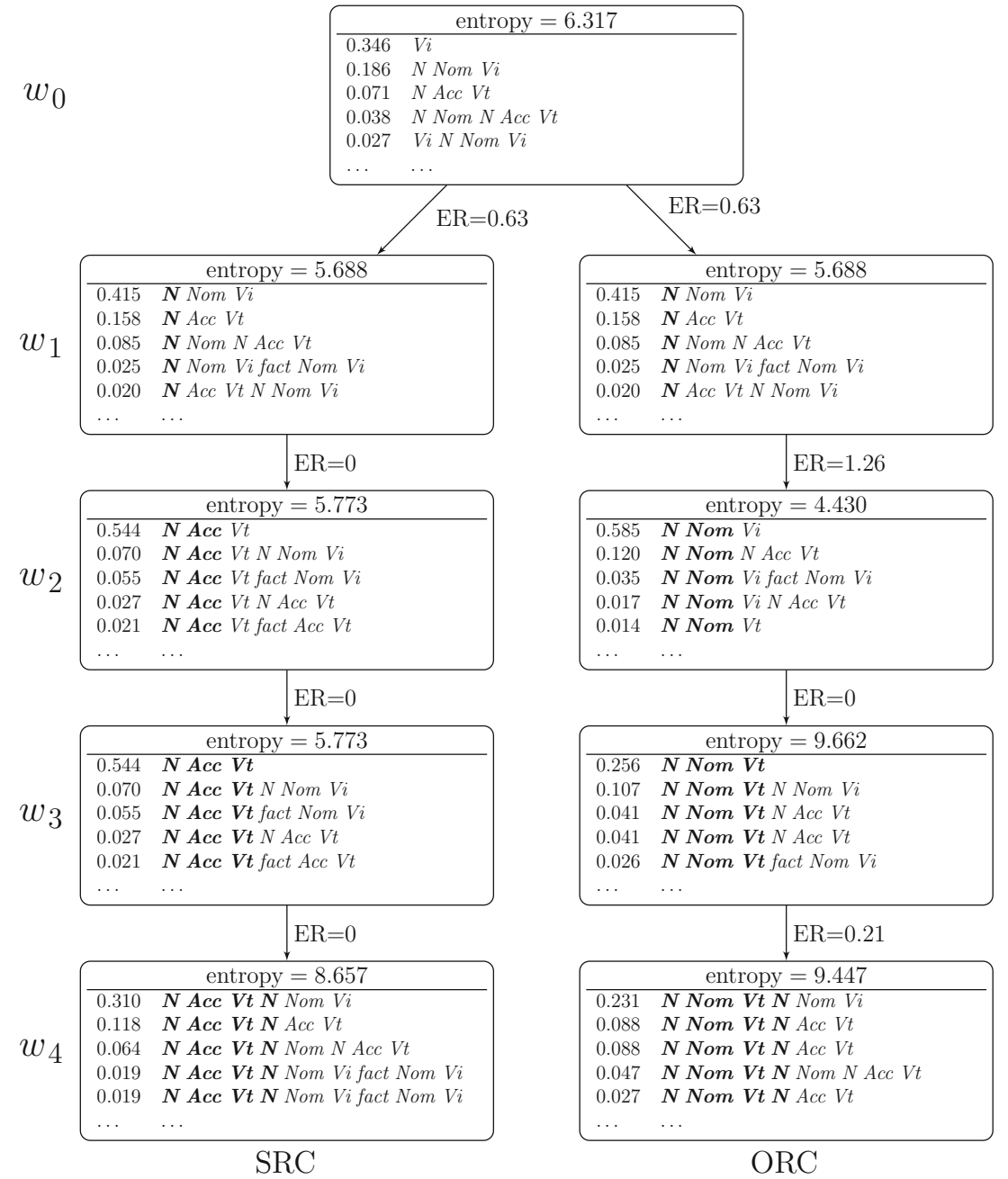

Fig. 9 Likely derivations and their conditional probabilities in Japanese RCs

the ORC-initial substring (9.662 bits) quantifies this claim. ${ }^{8}$ The entropy at the SRCinitial substring at $w_{3}$ is in fact so low that no Reduction happens at all in the transition from $w_{3}$ to $w_{4}$ in the SRC. This contributes to the Subject Advantage at the head noun $\left(w_{4}\right)$ in Japanese.

\footnotetext{
8 As in Korean, the additional remainders for the ORC-initial substring also contribute to its high entropy. However, the influence of ambiguous verb forms seems much stronger in processing Japanese RCs, as indicated by the highly asymmetric probability distributions of the SRC and ORC remainder-sets at the verb.
} 


\begin{tabular}{|c|c|c|}
\hline Rank & Prob & Remainder \\
\hline 1. & 0.544 & pro $\boldsymbol{N} \boldsymbol{A c c} V t$ \\
\hline 2. & 0.070 & {$\left[\begin{array}{llll}t & \boldsymbol{N} & \boldsymbol{A c c} & V t\end{array}\right] \quad \mathrm{N} N \mathrm{Nom} \mathrm{Vi}$} \\
\hline 3. & 0.055 & {$[$ pro $\mathbf{N}$ Acc $V t]$ fact Nom $\mathrm{Vi}$} \\
\hline 4. & 0.027 & pro $[t \boldsymbol{N} A c c \quad V t]] N A c c V t$ \\
\hline 5. & 0.021 & pro $[$ pro $\boldsymbol{N} \mathbf{A c c} V t]$ fact $A c c \quad V t$ \\
\hline 6. & 0.014 & 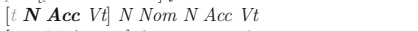 \\
\hline 7 . & 0.011 & {$\left[\begin{array}{llll}\text { pro } & \boldsymbol{N} \text { Acc } & V t\end{array}\right]$ fact Nom $N$ Acc $V t$} \\
\hline 8. & 0.010 & 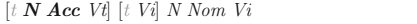 \\
\hline 9. & 0.008 & {$\left[\begin{array}{llll}t & \boldsymbol{N} & \boldsymbol{A c c} & V t\end{array}\right]\left[\begin{array}{ll}\text { pro } & \text { Vi }\end{array}\right]$ fact Nom $\mathrm{Vi}$} \\
\hline 10. & 0.004 & $\left[\begin{array}{llll}t & N & A c c & V t\end{array}\right] \quad N$ Nom $\left.V i\right]$ fact $N o m$ Vi \\
\hline 11. & 0.004 & {$\left[\begin{array}{llll}t & \boldsymbol{N} & \boldsymbol{A} c c & V t\end{array}\right]\left[\begin{array}{lll}N & \text { Nom } & V i\end{array}\right]$ fact $N o m$ Vi } \\
\hline 12. & 0.004 & pro $[t \quad \boldsymbol{N} A c c \quad V t]\left[\begin{array}{lll}t & V i\end{array}\right] N A c c \quad V t$ \\
\hline 13. & 0.003 & {$\left[\begin{array}{lllllll}t & \boldsymbol{N} & \boldsymbol{A} c c & V t\end{array}\right]\left[\begin{array}{llll}t & N & A c c & V t\end{array}\right] N$ Nom $V i$} \\
\hline 14. & 0.003 & $\left.\left[\begin{array}{llll}t & \boldsymbol{N} & \boldsymbol{A} c c & V t\end{array}\right] N A c c \quad V t\right] \quad N$ Nom $\mathrm{Vi}$ \\
\hline 15. & 0.003 & โ[pro $\boldsymbol{N} A$ Acc $V t]$ fact Nom Vi] fact Nom $V i$ \\
\hline 16. & 0.003 & pro $\left[\begin{array}{llll}t & \boldsymbol{N} & \boldsymbol{A} c \boldsymbol{c} & V t\end{array}\right]\left[\begin{array}{ll}\text { pro } & \text { Vi }\end{array}\right]$ fact $A c c \quad V t$ \\
\hline 17. & 0.003 & 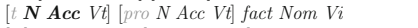 \\
\hline 18. & 0.003 & {$\left[\begin{array}{l}t \\
\text { pro }\end{array}\right.$} \\
\hline 19. & 0.003 & $\left.\left[\begin{array}{lll}\text { pro } & t \boldsymbol{N} \text { Acc } & V t\end{array}\right] \quad N A c c \quad V t\right]$ fact $N o m$ Vi \\
\hline 20. & 0.002 & 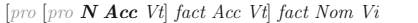 \\
\hline 21. & 0.002 & 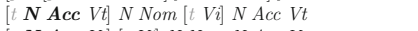 \\
\hline 22. & 0.002 & {$\left[\begin{array}{llll}t & \boldsymbol{N} & \boldsymbol{A} c c & V t\end{array}\right]\left[\begin{array}{ll}t & V i\end{array}\right] \mathrm{N} N o m \mathrm{NAcc} V t$} \\
\hline 23. & 0.002 & {$\left[\begin{array}{llll}t & \boldsymbol{N} & \boldsymbol{A} c c & V t\end{array}\right] \mathrm{N}$ Nom pro $V t$} \\
\hline 24. & 0.002 & {$\left[\begin{array}{llll}t & \boldsymbol{N} & \boldsymbol{A} c c & V t\end{array}\right] \mathbf{N}$ Nom $\left[\begin{array}{lll}\text { pro } & \text { Vi }\end{array}\right]$ fact $A c c$ Vt } \\
\hline 25. & 0.002 & {$\left[\begin{array}{llll}t & \boldsymbol{N} & \boldsymbol{A} c \boldsymbol{c} & V t\end{array}\right]\left[\begin{array}{lll}\text { pro } & V i\end{array}\right]$ fact $\mathrm{Nom} N \mathrm{Acc} V t$} \\
\hline 26. & 0.002 & 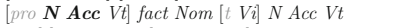 \\
\hline 27. & 0.002 & pro $\left[\left[\begin{array}{llll}t & \boldsymbol{N} & \boldsymbol{A} c c & V t\end{array}\right] \mathrm{N}\right.$ Nom $\left.\mathrm{Vi}\right]$ fact Acc $V t$ \\
\hline 28. & 0.002 & 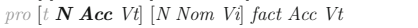 \\
\hline 29. & 0.001 & 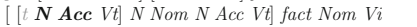 \\
\hline 30. & 0.001 & {$\left[\begin{array}{llll}t & \boldsymbol{N} & \boldsymbol{A} c c & V t\end{array}\right]\left[\begin{array}{lllll}N & \text { Nom } & N & A c c & V t\end{array}\right]$ fact $N o m$ Vi } \\
\hline 31. & 0.001 & {$\left[\begin{array}{llll}t & \boldsymbol{A} & \mathrm{Acc} & V t\end{array}\right]\left[\begin{array}{ll}t & V i\end{array}\right]\left[\begin{array}{ll}t & V i\end{array}\right] \mathrm{N} N o m \mathrm{Vi}$} \\
\hline 32. & 0.001 & 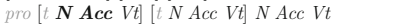 \\
\hline 33. & 0.001 & pro $\left[t\left[\begin{array}{lll}t & \boldsymbol{N} A c c & V t\end{array}\right] N A c c \quad V t\right] N A c c \quad V t$ \\
\hline 34. & 0.001 & {$[$ pro $\boldsymbol{N} \boldsymbol{A c c} V t]$ fact Nom pro $V t$} \\
\hline 35. & 0.001 & {$\left[\begin{array}{llll}t & \boldsymbol{N} & \boldsymbol{A} c c & V t\end{array}\right]\left[\begin{array}{lll}\text { pro } t & V t\end{array}\right]$ N Nom $V i$} \\
\hline 36. & 0.001 & 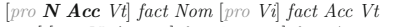 \\
\hline 37. & 0.001 & pro $\left[\left[\begin{array}{l}\text { pro } \mathbf{N} \boldsymbol{A c c} \\
\text { Vt } t]\end{array}\right]\right.$ fact Nom Vi $]$ fact Acc Vt \\
\hline 38. & 0.001 & 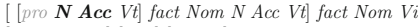 \\
\hline 39. & 0.001 & {$\left[\begin{array}{llll}t & \boldsymbol{N} & \mathrm{Acc} & V t\end{array}\right]\left[\begin{array}{ll}t & \mathrm{Vi}\end{array}\right]\left[\begin{array}{ll}\text { pro } & \mathrm{Vi}\end{array}\right]$ fact $\mathrm{Nom} \mathrm{Vi}$} \\
\hline 40. & 0.001 & pro $\left[\begin{array}{llll}t & N & A c c & V t\end{array}\right]\left[\begin{array}{llll}\text { pro } & N & A c c & V t\end{array}\right]$ fact $A c c$ Vt \\
\hline 41. & 0.001 & 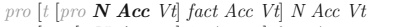 \\
\hline 42. & 0.001 & 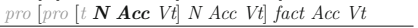 \\
\hline
\end{tabular}

(a) SRC-initial substring $N A c c$

\begin{tabular}{|c|c|c|}
\hline Rank & Prob & Remainder \\
\hline 1. & 0.585 & N Nom Vi \\
\hline 2. & 0.120 & N Nom $N A c c V t$ \\
\hline 3. & 0.035 & {$[N$ Nom $\mathrm{Vi}]$ fact Nom $\mathrm{Vi}$} \\
\hline 4. & 0.017 & $\boldsymbol{N}$ Nom $[t V i] N A c c V t$ \\
\hline 5. & 0.014 & $\boldsymbol{N} \mathbf{N o m}$ pro $V t$ \\
\hline 6. & 0.013 & pro $[\boldsymbol{N}$ Nom $V i]$ fact $A c c V t$ \\
\hline 7 . & 0.013 & $\boldsymbol{N}$ Nom $[$ pro Vi fact Acc Vt \\
\hline 8. & 0.012 & {$\left[\begin{array}{l}\boldsymbol{N} \text { Nom } N \text { Acc } \\
V t\end{array}\right]$ fact Nom Vi } \\
\hline 9. & 0.007 & {$[$ N Nom Vi] fact Nom $N$ Acc Vt } \\
\hline 10. & 0.007 & $N$ Nom $[N$ Nom Vi] fact Acc Vt \\
\hline 11. & 0.006 & $N$ Nom $[t N A c c \quad V t] \quad N A c c \quad V t$ \\
\hline 12. & 0.006 & {$[N$ Nom $t$ Vt] $N$ Nom $V i$} \\
\hline 13. & 0.005 & pro $[\boldsymbol{N}$ Nom $N A c c \quad V t]$ fact $A c c V t$ \\
\hline 14. & 0.005 & $\boldsymbol{N}$ Nom $[$ pro $N A c c$ Vt fact Acc $V t$ \\
\hline 15. & 0.002 & {$[\boldsymbol{N}$ Nom $N$ Acc $V t]$ fact Nom $N$ Acc Vt } \\
\hline 16. & 0.002 & $\boldsymbol{N} \mathbf{N o m}[N$ Nom $N$ Acc $V t]$ fact $A c c V t$ \\
\hline 17. & 0.002 & $\boldsymbol{N}$ Nom $[t \quad V i][t$ Vi $] N A c c V t$ \\
\hline 18. & 0.002 & pro $[\boldsymbol{N}$ Nom $t V t] N A c c V t$ \\
\hline 19. & 0.002 & $\boldsymbol{N} \mathbf{N o m}[$ pro $t V t] N A c c V t$ \\
\hline 20. & 0.002 & {$[[\boldsymbol{N}$ Nom Vi] fact Nom Vi] fact Nom Vi } \\
\hline 21. & 0.002 & $\boldsymbol{N}$ Nom $\left[t\right.$ Vi $\left[\begin{array}{lll}\text { pro } & V i\end{array}\right]$ fact Acc $V t$ \\
\hline 22. & 0.002 & {$[N$ Nom $[t \quad V i] N A c c$ Vt $]$ fact Nom $V i$} \\
\hline 23. & 0.002 & {$[t[\boldsymbol{N} \mathbf{N o m}$ Vi $]$ fact $A c c \quad V t] N$ Nom $V i$} \\
\hline 24. & 0.001 & {$[\mathbf{N}$ Nom pro $V t]$ fact Nom $\mathrm{Vi}$} \\
\hline 25. & 0.001 & {$[$ pro $[\boldsymbol{N}$ Nom Vi] fact Acc Vt] fact Nom Vi } \\
\hline 26. & 0.001 & {$[\boldsymbol{N}$ Nom $[$ pro $V i]$ fact Acc Vt fact Nom Vi } \\
\hline 27. & 0.001 & {$[$ N Nom $t V t] N$ Nom $N$ Acc Vt } \\
\hline 28. & 0.001 & $\boldsymbol{N}$ Nom $[N$ Nom $t V t] N A c c V t$ \\
\hline 29. & 0.001 & {$[N$ Nom Vi] fact Nom $[t$ Vi $]$ NAcc Vt } \\
\hline 30. & 0.001 & $\boldsymbol{N}$ Nom $\left[t\right.$ Vi $\left[{ }^{N}\right.$ Nom $\left.V i\right]$ fact Acc $V t$ \\
\hline 31. & 0.001 & N Nom $[[t$ Vi $]$ N Nom $V i]$ fact Acc $V t$ \\
\hline
\end{tabular}

(b) ORC-initial substring $N$ Nom

Fig. 10 Expanded derivation lists $(p>0.001)$ at the second words in Japanese RCs

\subsection{Chinese}

The true processing difficulty profile of Chinese RCs has been tough to pin down. One of the factors that confounds research on SRCs and ORCs in this language is the presence of temporary ambiguities. A variety of syntactic alternatives are compatible with the initial substrings of these constructions. For example, the SRC-initial substring (Vt N ...) can also be understood as a pro-dropped matrix clause. Given that subject pro-drop in Chinese is extremely frequent, ${ }^{9}$ this possibility must be taken into account. ORCs, too, are plagued by troublesome temporary ambiguities. For instance, the ORCinitial substring $(\mathrm{N} \mathrm{Vt} \mathrm{...)} \mathrm{may} \mathrm{also} \mathrm{continue} \mathrm{as} \mathrm{a} \mathrm{matrix} \mathrm{clause.} \mathrm{If} \mathrm{readers} \mathrm{have} \mathrm{a} \mathrm{strong}$ expectation for this structure, they may not realize they have read an ORC. Moreover, when an ORC modifies the matrix object, the initial substring ( $\mathrm{N} \mathrm{Vt}[\mathrm{N} \mathrm{Vt} \mathrm{\ldots .)} \mathrm{can}$ trigger a garden-path effect of reanalysis from a matrix clause to an RC (Lin and Bever 2006, 2011).

\footnotetext{
9 In our Chinese Treebank search (see Appendix 1(c) for more results), we find that there are even more omitted arguments (6385) than bare nouns (3830) in subject position.
} 


\begin{tabular}{|lll|}
\hline Probability & Remainder & Type \\
\hline 0.544 & pro $\boldsymbol{N} \boldsymbol{A} \boldsymbol{c c} \boldsymbol{V} \boldsymbol{t}$ & simplex SOV with Sbj-pro \\
0.070 & {$[$ t $\boldsymbol{N} \boldsymbol{A c c} \boldsymbol{V} \boldsymbol{t}] \boldsymbol{N}$ Nom $V i$} & SRC \\
0.055 & {$[$ pro $\boldsymbol{N} \boldsymbol{A c c} \boldsymbol{V} \boldsymbol{t}]$ fact $N o m$ Vi } & NCC with Sbj-pro \\
0.027 & pro $[$ t $\boldsymbol{A} \boldsymbol{A c c} \boldsymbol{V} \boldsymbol{t}]$ N Acc $V t$ & SRC \\
0.021 & pro $[$ pro $\boldsymbol{N} \boldsymbol{A} \boldsymbol{c c} \boldsymbol{V} \boldsymbol{t}]$ fact $A c c$ Vt & NCC with Sbj-pro \\
$\ldots$ & $\ldots$ & $\ldots$ \\
\hline Entropy $=5.773$ & \\
\hline
\end{tabular}

(a) SRC prefix $N$ Acc $V t$

\begin{tabular}{|lll|}
\hline Probability & Remainder & Type \\
\hline 0.256 & {$[\boldsymbol{N}$ Nom pro $\boldsymbol{V t}]$} & Simplex SOV with Obj-pro \\
0.107 & {$[\boldsymbol{N}$ Nom $t \boldsymbol{V} \boldsymbol{t}]$ Nom Vi } & ORC \\
0.041 & pro $[\boldsymbol{N}$ Nom $t \boldsymbol{V t}] N$ Acc Vt & ORC \\
0.041 & $\boldsymbol{N}$ Nom $[$ pro $t \boldsymbol{V t}]$ NAcc Vt & ORC with Sbj-pro \\
0.026 & {$[\boldsymbol{N}$ Nom pro $\boldsymbol{V t}]$ fact Nom Vi } & NCC with Obj-pro \\
$\ldots$ & $\ldots$ & $\ldots$ \\
\hline Entropy $=9.662$ & \\
\hline
\end{tabular}

(b) ORC prefix $N$ Nom $V t$

Fig. 11 Possible remainders at the third word for Japanese RCs

In order to test whether the Subject Advantage indeed exists in Chinese RCs, Jäger et al. (in press) used an experimental design where RC-initial substrings are disambiguated from matrix clauses. The disambiguation is accomplished using extra words that help guide readers towards some RC interpretation while still leaving the specific gap site unspecified.

a. "Disambiguated" Chinese SRC

na ge zuotian [ $\mathrm{e}_{i}$ yaoqing fuhao de $]$ guanyuan $_{i}$ that.Dem $\mathrm{Cl}$ yesterday.Time [ $t$ invite. Vt tycoon.N DE ] official.N

'the official who invited the tycoon yesterday'

b. "Disambiguated" Chinese ORC

na ge zuotian [ fuhao yaoqing $\mathrm{e}_{i}$ de ] guanyuan $_{i}$ that.Dem $\mathrm{Cl}$ yesterday.Time [ tycoon. $\mathrm{N}$ invite. $\mathrm{Vt} t \mathrm{DE}$ ] official. $\mathrm{N}$

'the official who the tycoon invited yesterday'

In Example (12), the sentence-initial demonstrative-classifier combination "na-ge" encourages readers to expect a noun phrase. However, the following word is a temporal phrase "yesterday" which has to be attached to a verb phrase. This design therefore leads the reader to only foresee an upcoming RC-modified noun phrase by ruling out the pro-drop analysis. Jäger et al. (in press) used these "disambiguated" RC stimuli in both self-paced reading and eye-tracking experiments. They reported that SRCs are consistently read faster than ORCs in the RC region ( $V t N$ or $N V t$, respectively) and at the head noun. A Subject Advantage was also found after the head noun, potentially a spillover effect from previous regions. 

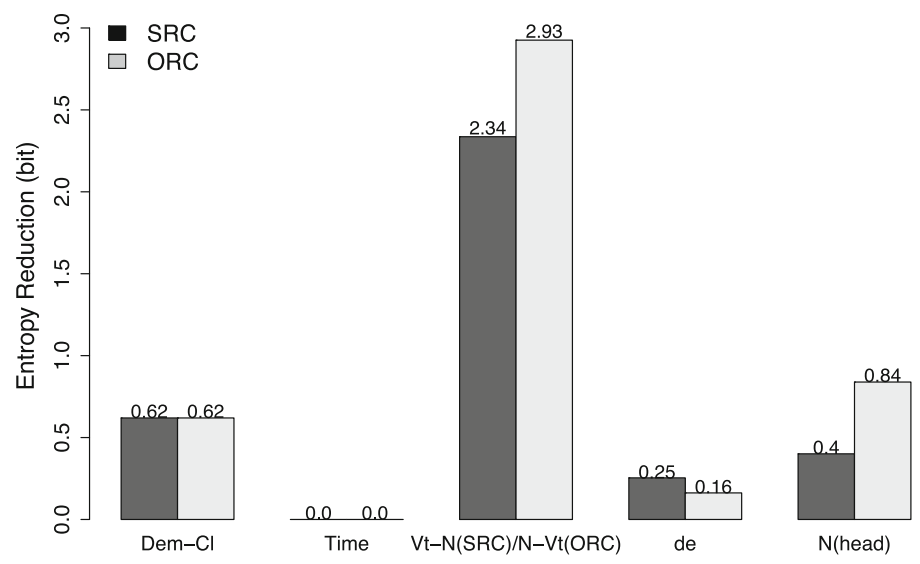

Fig. 12 ER predictions for "disambiguated" Chinese RCs

Entropy Reduction derives a Subject Advantage in Chinese RCs as shown in Fig. 12. ORCs are correctly categorized as harder to process, in both the RC and head noun regions.

Figure 13 illustrates how the Subject Advantage is predicted in Chinese RCs regionby-region. Because the word order of SRCs $(V t N)$ is not the same as that of ORCs $(N V t)$ in Chinese, we collapse the two words in the RC region. After processing this $\mathrm{RC}$ region, entropy reduces by 2.34 bits in the SRC case and 2.93 bits in the ORC case. Looking at the boxes in the "RC region" row, we focus on the two initial substrings Dem Cl Time Vt $\mathrm{N}$ and Dem Cl Time $\mathrm{N}$ Vt.

The greater Entropy Reduction in the ORC case as compared to the SRC case quantifies the intuitive idea that more comprehension work is called for in the RC region of one item as compared to the other. This numerical contrast reflects the fact that, by the end of the $\mathrm{RC}$ region, the $\mathrm{ORC}$ parser state is more organized than the corresponding SRC state. Table 5 quantifies this degree of organization by counting the remainders at various probability thresholds. While both initial substrings start from the same uncertainty level (7.448 bits), the ORC-initial substring goes farther in reducing this ambiguity. In other words, more work has been done.

Earlier modeling work on Chinese RCs using Surprisal did not derive the Subject Advantage at the RC head noun (Z. Chen et al. 2012); however, the present work with Entropy Reduction does. Examining the pre-head syntactic remainders in Fig. 14, the grammar defines contrasting probabilities for headlessness in SRC vs ORC contexts. In ORCs, as shown in the bottom left box, there is at least a $20.8 \%$ chance that the initial substring will continue as a headless RC. On the other hand, it is less likely $(5.6 \%)$ that an SRC-initial substring will be headless (top left box). The informationvalue of the headlessness question itself is different across the two constructions, and this contributes to the prediction of a Subject Advantage at the head word in Chinese. 


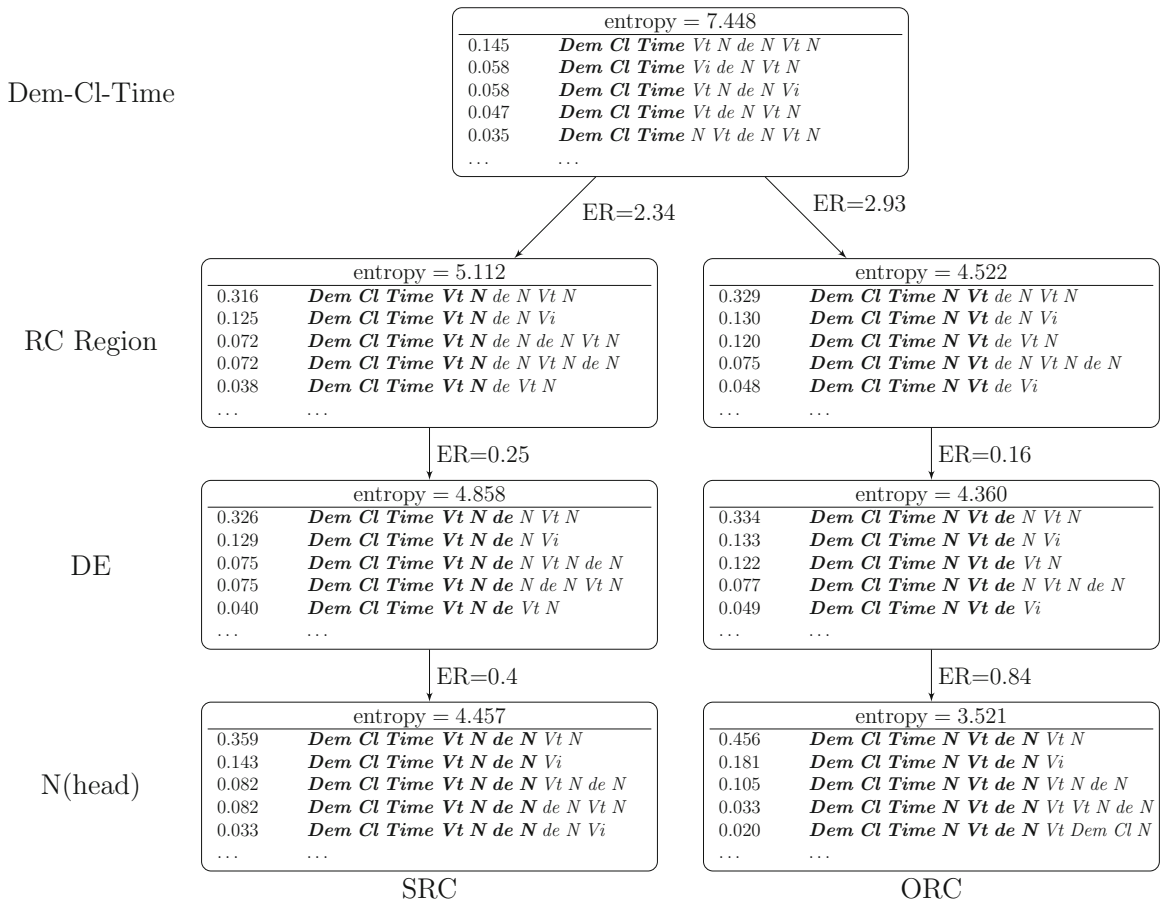

Fig. 13 Derivations and their conditional probabilities in "disambiguated" Chinese RCs

Table 5 More peaked probability distribution of remainders leads to lower uncertainty level in ORCs. Starting from the same entropy level (first row of Fig. 13) but arriving at a lower "destination" level (second row of Fig. 13) in just the ORC yields the prediction of greater processing effort via the Entropy Reduction hypothesis

\begin{tabular}{|c|c|c|c|c|}
\hline Probability & Type & No. of Remainders & Total Probability & Uncertainty \\
\hline \multirow{2}{*}{0.01} & SRC & 10 & 0.722 & High \\
& ORC & 8 & 0.768 & Low \\
\hline \multirow{2}{*}{0.001} & SRC & 64 & 0.881 & High \\
& ORC & 54 & 0.908 & Low \\
\hline
\end{tabular}

\section{Conclusion}

Processing asymmetries across relative clause types can be understood as differential degrees of confusion about sentence structure. On the one hand, this idea has a certain intuitive obviousness about it: a harder information-processing problem ought to be associated with greater observable processing difficulty. But it is not a foregone conclusion. It could have been the case that the sentence structures motivated by leading syntactic analyses do not derive these "harder" vs "easier" information processing problems in just the cases where human readers seem to have trouble. But in fact, as Section 6 details, they do. 


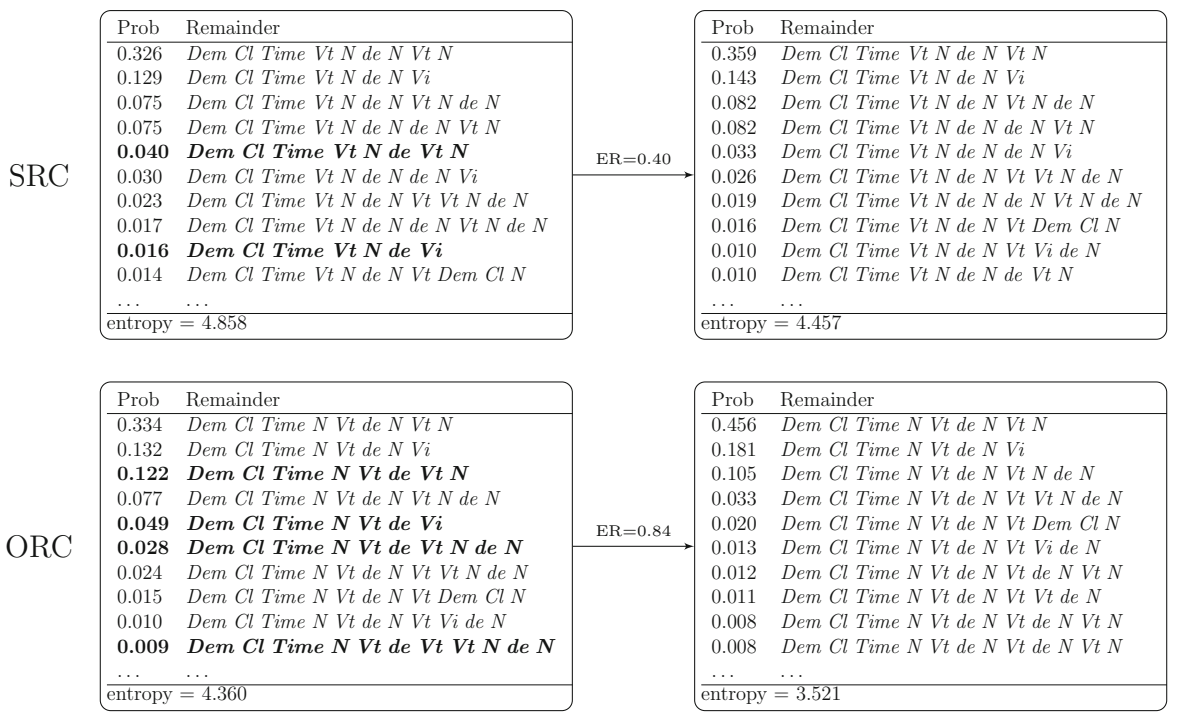

Fig. 14 ER at the head noun in Chinese RCs; detail of last two rows in Fig. 13. Here, boldface highlights expectations that the RC will be headless

By suggesting that human listeners are information-processors subject to information theory, this methodology revives the approach of psychologists like Hick (1952), Attneave (1959) and Garner (1962). What differs in the present application of information theory is the use of generative grammars to specify the sentence-structural expectations that listeners have. The conditional probabilities in diagrams like Fig. 5 are calculated over grammatical alternatives, not just single successor words. In this regard our method integrates both information-processing psychology and generative grammar.

This integration articulates the view that all humans are bound by the same kinds of processing limitations. Where the distribution on grammatical alternatives goes from being flatter to being more peaked, we should expect slower reading times. Such a limitation may perhaps reflect something deep about cognition, something that constrains both humans and machines. However, the precise implications of this limit depend crucially on the grammar of particular languages.

Acknowledgments The research reported in this article was supported by NSF CAREER award number 0741666. JY and JBW were partially supported by a lab grant (AKS-2011-AAA-2103) from the Academy of Korean Studies.

Open Access This article is distributed under the terms of the Creative Commons Attribution License which permits any use, distribution, and reproduction in any medium, provided the original author(s) and the source are credited. 


\section{Appendix 1: Attestation counts}

(a) Korean

Korean Treebank 2.0 (Han et al. 2002) was used to obtain an estimation of the frequencies of the relevant structures in Korean. The texts of the corpus are a selection of Korean Press Agency news articles in 2000, consisting of 5,010 sentences and 132,040 words. The weight parameters for the MG presented in example 6 on page 123 were derived from the attestation counts given below in Table 6.

Table 6 Korean attestation counts

\begin{tabular}{|l|l|l|l|r|}
\hline Clause Type & Subject & Object & Verb & Count \\
\hline Matrix Clause & overt & overt & transitive & 24 \\
& overt & pro & transitive & 2 \\
& pro & overt & transitive & 31 \\
& pro & pro & transitive & 0 \\
& overt & - & intransitive & 436 \\
& pro & - & intransitive & 30 \\
\hline Complement Clause & overt & overt & transitive & 92 \\
& overt & pro & transitive & 5 \\
& pro & overt & transitive & 401 \\
& pro & pro & transitive & 12 \\
& overt & - & intransitive & 282 \\
& pro & - & intransitive & 110 \\
\hline Relative Clause (SRC) & gap & overt & transitive & 467 \\
& gap & pro & transitive & 4 \\
& gap & - & intransitive & 559 \\
\hline Relative Clause (ORC) & overt & gap & transitive & 120 \\
& pro & gap & transitive & 10 \\
\hline
\end{tabular}

(b) Japanese

In the case of Japanese, Kyoto Corpus 4.0 (Kurohashi and Nagao 2003) was used. The texts of the corpus are a selection of Mainichi Newspaper articles from 1995, consisting of 5,447 sentences and 161,028 words. Although the Kyoto Corpus is not a treebank, it does provide a rich set of part-of-speech tags that we used to carry out a corpus study analogous to the Korean study discussed above. In particular, all predicates are annotated with information about their arguments, including the location of the argument and its syntactic type (such as nominative or accusative). Although Japanese RCs are prenominal, the canonical word order places the verb at the end of the clause, after all of its arguments. Consequently, if a verb is followed by its nominative or accusative argument, the clause that ends with the verb is an SRC 
or ORC, respectively. If a verb comes after all its arguments but still precedes some other noun, then the clause that ends with the verb is a noun complement clause. The frequencies of the same parameters as in Korean were derived from the counts in Table 7.

Table 7 Japanese attestation counts

\begin{tabular}{|l|l|l|l|r|}
\hline Clause Type & Subject & Object & Verb & Count \\
\hline Matrix Clause & overt & overt & transitive & 125 \\
& overt & pro & transitive & 7 \\
& pro & overt & transitive & 329 \\
& pro & pro & transitive & 32 \\
& overt & - & intransitive & 691 \\
& pro & - & intransitive & 487 \\
\hline Complement Clause & overt & overt & transitive & 149 \\
& overt & pro & transitive & 4 \\
& pro & overt & transitive & 323 \\
& pro & pro & transitive & 25 \\
& overt & - & intransitive & 463 \\
& pro & - & intransitive & 200 \\
\hline Relative Clause (SRC) & gap & overt & transitive & 537 \\
& gap & pro & transitive & 20 \\
& gap & - & intransitive & 854 \\
\hline Relative Clause (ORC) & overt & gap & transitive & 116 \\
& pro & gap & transitive & 102 \\
\hline
\end{tabular}

\section{(c) Chinese}

We obtain attestation counts from Chinese Treebank 7 (Xue et al. 2005) which contains 51,447 fully parsed sentences or 1,196,329 words. These yield the weights shown below in Table 8 . Note that the "disambiguated" RCs shown in example 12 on page 136 motivate a somewhat richer set of choice points in the formal grammar fragment, which obligates us to estimate weights for a longer list of parameters than in Korean or Japanese. 
Table 8 Chinese attestation counts

\begin{tabular}{|l|r|}
\hline noun with a demonstrative modifier & 2916 \\
complex NP with a demonstrative modifier & 345 \\
\hline noun in argument position & 8133 \\
complex NP in argument position & 2316 \\
possessive phrase in argument position & 1866 \\
\hline headful SRC & 2281 \\
headless SRC & 280 \\
headful ORC & 830 \\
headless ORC & 304 \\
\hline noun in subject position & 3830 \\
noun with a demonstrative modifier in subject position & 167 \\
pro in subject position & 6385 \\
noun in object position & 3766 \\
noun with a demonstrative modifier in object position & 123 \\
pro in object position & 2 \\
\hline subject pro with transitive verb & 5054 \\
subject pro with intransitive verb & 1331 \\
subject NP with transitive verb & 17250 \\
subject NP with intransitive verb & 4377 \\
\hline noun as ORC subject & 185 \\
noun with a demonstrative modifier as ORC subject & 12 \\
pro as ORC subject & 162 \\
\hline matrix modified by temporal adjunct & 343 \\
matrix not modified by temporal adjunct & 16852 \\
\hline SRC not modified by temporal adjunct & 2532 \\
ORC not modified by temporal adjunct & 1124 \\
RC modified by temporal adjunct & 39 \\
\hline relative clause & 3695 \\
complement clause & \\
\hline
\end{tabular}

\section{Appendix 2: A note on the infinity of possible remainders}

References to dozens of possible sentence-remainders in, for example, Table 5 or Fig. 7 , might prompt the question of whether our account assumes some degree of parallelism in the parsing process. It is true that, for example, (the probability of) the $100^{\text {th }}$-best possible remainder plays a role in determining the predictions of the Entropy Reduction Hypothesis, but this does not entail any algorithmic claim that the comprehender in fact proceeds by considering each of the top 100 derivations one by one.

Instead of a processing algorithm, the ER complexity metric rather models a comprehender's intermediate mental state at a particular position in a sentence using a grammar. Section 5 dubs this sort of object an "intersection grammar." Such a grammar is a finite object that characterizes the set of possible sentence-remainders, which is in many cases an infinite set. This characterization is precisely analogous to the way 


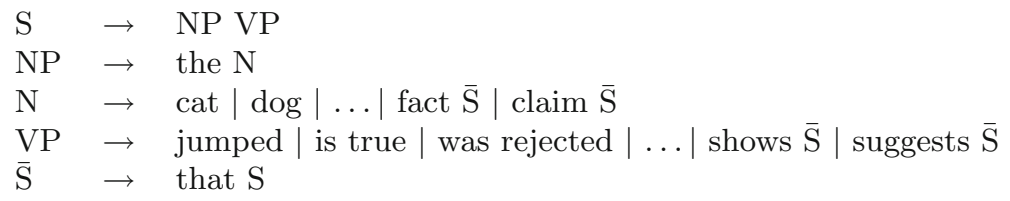

Fig. 15 A simple grammar for an imagined language

that generative grammars are ordinarily used to characterize presumably infinite sets of well-formed $u n$-remaindered, full sentences. ${ }^{10}$

The important idea is that when we report, for example, an entropy value of 5.773 after encountering the initial substring $N A c c$ in Fig. 9, this value is a property of the intersection grammar that finitely characterizes the comprehender's mental state at that point; we can ask the question of what this entropy value is in much the same way that we can ask, say, how many rules are in the grammar, or what the greatest number of symbols on the right hand side of a single rule is. (These are other conceivable, though perhaps not so well-motivated, linking hypotheses one might consider using to connect intersection grammars to behavioral predictions.) In order to gain some understanding of the content of a remainder grammar, it is useful to consider some of the infinitely many derivations that it licenses, and this is the purpose of derivation lists that appear throughout Section 6. These lists help to understand the implications of the remainder grammars, but it is the remainder grammars' uncertainty properties, and not the lists themselves that we offer as a cognitive account of comprehenders' intermediate mental states.

To illustrate this point, consider the grammar in Fig. 15. This finite object derives infinitely many sentences such as the following (where the brackets are added just for readability).

(13) a. The fact that [John met Mary] shows that [the cat jumped]

b. The fact that [the claim that [the cat jumped] was rejected] suggests that [the report that [John met Mary] was true]

c. $\ldots$

This grammar licenses an infinity of derivations because it contains "loops." For example, an $\mathrm{S}$ can contain a VP, which can contain an $\overline{\mathrm{S}}$, which can contain an $\mathrm{S}$, as illustrated in Fig. 16a. There is also a second, longer, loop, consisting of four nonterminals, shown in Fig. 16 b.

Consider now a comprehender using this grammar to parse a sentence, at the point where only the initial substring The fact that John met Mary shows has been encountered. Our methodology characterizes this comprehender's mental state at this point by an interion grammar, a modified version of the grammar in Fig. 15 that derives only sentences consistent with this initial substring. Clearly there are an infinite number

\footnotetext{
10 In the special case where we consider the comprehender's mental state at the beginning of a sentence, i.e. where the initial substring encountered so far is the empty string, these two ideas reduce to the same thing: the possible remainders consistent with this empty initial substring are precisely the well-formed sentences of the language.
} 
Fig. 16 The two loops present in the grammar in Fig. 15

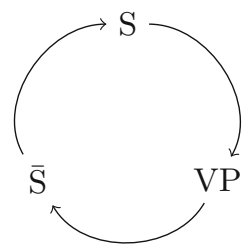

(a) One loop

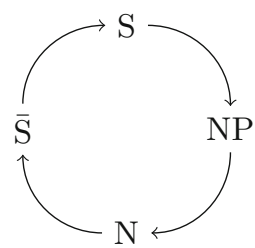

(b) Another loop

of such sentences, since they have the form The fact that John met Mary shows that $S$; hence the intersection grammar must necessarily contain at least one loop. Note that this is true even though there are an infinite number of other sentences that are no longer candidates because they are not consistent with the heard initial substring, but which are derivable using the grammar in Fig. 15, since the main clause subject can contain unboundedly many embeddings. More specifically, then, the remainder grammar will have in effect the same two loops shown in Fig. 16, although the ways these loops can be reached are more restricted than is the case for the grammar in Fig. 15.

The import of this example for present purposes is this: just as the infinite list of sentences in this imagined language can be characterized by the finite grammar in Fig. 15, and hence by a certain finite mental state, so can the infinite list of sentences consistent with the relevant initial substring. In order to gain some intuitive understanding of what is characterized by such a finite mental state, it is often useful to "unroll" some of the licensed derivations for inspection; this is what we are doing when we look at (13) to gain some understanding of the grammar in Fig. 15, and the larger lists in Section 6 were used to similarly gain some understanding of certain intersection grammars. These lists themselves, however, play no role in the theory we propose. Just as a competent speaker is usually hypothesized to bear a certain mental relationship to a grammar such as the one in Fig. 15, rather than any particular elements of or subsets of lists like (13), in our view a comprehender bears a certain mental relationship to an intersection grammar (which, in turn, has an entropy), rather than any particular elements of or subsets of lists like the ones presented in preceding subsections.

\section{Appendix 3: Entropy reduction and information theory}

The reduction in entropy of grammatical derivations brought about by observing (or lengthening) an initial substring represents a particular way of characterizing information gain. This particular way is written out as the difference of two entropies in 14 below. In this definition, $X$ is a random variable over derivations. $Y=y$ denotes the outcome of a related random variable such as an initial substring of the yield.

$$
I(X ; y)=H(X)-H(X \mid y)
$$

Blachman (1968) compares the information measure in 14 to an alternative measure given below in 15 . 


$$
J(X ; y)=\sum_{x} p(x \mid y) \log \left(\frac{p(x \mid y)}{p(x)}\right)
$$

The second measure, $J$, leads to surprisal in the sense of Hale (2001) via reasoning analogous to that presented in Section 2.1 of Levy (2008). The first measure, $I$, leads to Entropy Reduction. As Blachman points out, both notions of information gain have as their expectation the mutual information of $X$ and $Y$. However, he goes on to show that they are not equivalent. For instance, $I$ can be negative whereas $J$ cannot. On the other hand, $I$ is additive while $J$ is not.

What this shows is that there is no one "official" way to apply information theory to human language processing. These definitions are theoretical postulations that are useful to the extent that they lead to greater insight into the phenomena under study.

\section{References}

Attneave, Fred. 1959. Applications of Information Theory to Psychology: A summary of basic concepts, methods and results, Oxford: Holt, Rinehart and Winston.

Bar-Hillel, Yehoshua, Micha Perles, and Eliyahu Shamir. 1964. On formal properties of simple phrase structure grammars. In Language and Information: Selected Essays on their Theory and Application, Reading, Massachusetts: Addison-Wesley, Chap 9, 116-150.

Berwick, Robert, and Samuel Epstein. 1995. On the Convergence of 'Minimalist' Syntax and Categorial Grammar. In: Algebraic methods in language processing : proceedings of the tenth Twente Workshop on Language Technology joint with first AMAST Workshop on Language Processing. ed. A. Nijholt.

Bever, Thomas. 1970. The cognitive basis for linguistic structures. In Cognition and the development of language, ed. J. Hayes, 279-360. John Wiley.

Blachman, Nelson. 1968. The amount of information that $y$ gives about X. IEEE Transactions on Information Theory IT 14(1):27-31.

Brame, Michael. 1967. A new analysis of the relative clause: evidence for an interpretive theory. Unpublished manuscript.

Chen, Baoguo, Aihua Ning, Hongyan Bi, and Susan Dunlap. 2008. Chinese subject-relative clauses are more difficult to process than the object-relative clauses. Acta Psychologica 129(1):61-65.

Chen, Zhong. 2014. Animacy in sentence processing across languages: an information-theoretical perspective. Ph.D. dissertation, Cornell University.

Chen, Zhong, Kyle Grove and John Hale. 2012. Structural expectations in Chinese relative clause comprehension. In Proceedings of the 29th West Coast Conference on Formal Linguistics (WCCFL-29), ed. J. Choi, E. A. Hogue, J. Punske, D. Tat, J. Schertz, and A. Trueman, 29-37, Somerville, MA: Cascadilla Proceedings Project.

Cherry, Colin. 1961. On human communication: a review, a survey, and a criticism, 2nd edn. New York: Science Ed.

Chomsky, Noam. 1993. A minimalist program for linguistic theory. In The view from building 20: essays in linguistics in honor of Sylvain Bromberger, Current studies in linguistics, Vol 24, 1-52. Cambridge, Mass.: MIT Press.

Chomsky, Noam. 1995. The Minimalist Program. Cambridge, Mass.: MIT Press.

Frank, Stefan. 2013. Uncertainty reduction as a measure of cognitive load in sentence comprehension. Topics in Cognitive Science 5(3):475-494. doi:10.1111/tops.12025.

Frauenfelder, Uli, Juan Segui, and Jacques Mehler. 1980. Monitoring around the relative clause. Journal of Verbal Learning and Verbal Behavior 19:328-337.

Frazier, Lyn. 1987. Syntactic processing: Evidence from Dutch. Natural Language \& Linguistic Theory 5(4):519-559.

Garner, Wendell. 1962. Uncertainty and structure as psychological concepts. New York: Wiley.

Gibson, Edward. 2000. Dependency locality theory: A distance-based theory of linguistic complexity. In Image, Language, brain: Papers from the First Mind Articulation Project Symposium, ed. A. Marantz, Y. Miyashita, and W. O’Neil. Cambridge, Mass: MIT Press. 
Gibson, Edward and Hsiao-Hung Iris Wu. 2013. Processing Chinese relative clauses in context. Language and Cognitive Processes 28(1-2):125-155.

Grenander, Ulf. 1967. Syntax-controlled probabilities. Technical Report. Providence, RI: Brown University Division of Applied Mathematics.

Guillaumin, Matthieu. 2005. Conversations between mildly context-sensitive grammars. Internship report, Ecole Normale Superieure and UCLA.

Hale, John. 2001. A probabilistic Earley parser as a psycholinguistic model. In Proceedings of the $2 n d$ NAACL, Pittsburgh, PA.

Hale, John. 2003. The information conveyed by words in sentences. Journal of Psycholinguistic Research 32(2):101-123.

Hale, John. 2006. Uncertainty about the rest of the sentence. Cognitive Science 30(4):643-672.

Han, Chung-hye, Na-Rae Han, Eon-Suk Ko, and Martha Palmer. 2002. Development and Evaluation of a Korean Treebank and its Application to NLP. Language and Information 6(1):123-138.

Han, Chung-hye and Jong-Bok Kim. 2004. Are there "double relative clauses" in Korean? Linguistic Inquiry 35(2):315-337.

Harkema, Henk. 2001. Parsing minimalist grammars. Ph.D. Dissertation, University of California, Los Angeles.

Harris, Theodore. 1963. The Theory of Branching Processes. Springer-Verlag.

Hawkins, John. 2004. Efficiency and Complexity in Grammars. Oxford University Press.

Hick, William. 1952. On the rate of gain of information. Quarterly Journal of Experimental Psychology 4 (1):11-26.

Hirose, Yuki. 2009. Processing relative clauses in Japanese: coping with multiple ambiguities. In The Handbook of East Asian Psycholinguistics, Vol. II, Chapter 35, 264-269. Cambridge: Cambridge University Press.

Hoshi, Koji. 1995. Structural and interpretive aspects of head-internal and head-external relative Structural and interpretive aspects of head-internal and head-external relative clauses. Ph.D. dissertation, University of Rochester.

Hsiao, Franny, and Edward Gibson. 2003. Processing relative clauses in Chinese. Cognition 90:3-27.

Hsiao, Yaling, Jinman Li, and Maryellen MacDonald. 2014. Ambiguity affects Mandarin relative clause processing. In The 27th annual CUNY conference on human sentence processing, Columbus, $\mathrm{OH}$. The Ohio State University.

Huang, C.-T. James, Yen-Hui Audrey Li, and Yafei Li. 2009. The Syntax of Chinese. Cambridge University Press.

Ishii, Yasuo. 1991. Operators and empty categories in Japanese. Ph.D. dissertation, University of Connecticut.

Ishizuka, Tomoko. 2005. Processing relative clauses in Japanese. In Papers in Psycholinguistics 2, UCLA Working Papers in Linguistics, ed. R. Okabe and K. Nielsen, Volume 13, 135-157, Los Angeles: UCLA Linguistics Department, Los Angeles.

Ishizuka, Tomoko, Kentaro Nakatani, and Edward Gibson. 2003. Relative clause extraction complexity in Japanese. Poster presented at the 16th Annual CUNY Conference on Human Sentence Processing.

Jäger, Lena, Zhong Chen, Qiang Li, Chien-Jer Charles Lin, and Shravan Vasishth. (in press). The subjectrelative advantage in Chinese: Evidence for expectation-based processing. Journal of Memory and Language.

Jurafsky, Daniel. 1996. A probabilistic model of lexical and syntactic access and disambiguation. Cognition 20:137-194.

Just, Marcel, Patricia Carpenter, Timothy Keller, William Eddy, and Keith Thulborn. 1996. Brain Activation Modulated by Sentence Comprehension. Science 274(5284):114.

Kaplan, Tamar, and John Whitman. 1995. The Category of Relative Clauses in Japanese, with Reference to Korean. Journal of East Asian Linguistics 4(1):29-58.

Kayne, Richard S. 1994. The Antisymmetry of Syntax. Cambridge, MA: MIT Press.

Keenan, Edward, and Bernard Comrie. 1977. Noun phrase accessibility and universal grammar. Linguistic Inquiry 8(1):63-99.

Keenan, Edward and Sarah Hawkins. 1987. The psychological validity of the Accessibility Hierarchy. In Universal Grammar: 15 Essays, ed. E. Keenan, 60-85. London: Croom Helm.

King, Jonathan and Marcel Just. 1991. Individual differences in syntactic processing: The role of working memory. Journal of Memory and Language 30(5):580-602. 
King, Jonathan and Marta Kutas. 1995. Who did what and when? Using word- and clause-level ERPS to monitor working memory usage in reading. Journal of Cognitive Neuroscience 7(3):376-395.

Kurohashi, Sadao and Makoto Nagao. 2003. Building a Japanese Parsed Corpus. In Treebanks, Text, Speech and Language Technology, ed. A. Abeillé and N. Ide Vol. 20, 249-260, Springer Netherlands. doi:10. 1007/978-94-010-0201-1_14.

Kwon, Nayoung, Yoonhyoung Lee, Peter C. Gordon, Robert Kluender, and Maria Polinsky. 2010. Cognitive and linguistic factors affecting subject/object asymmetry: An eye-tracking study of pre-nominal relative clauses in Korean. Language 86(3):546-582.

Kwon, Nayoung, Maria Polinsky, and Robert Kluender. 2006. Subject Preference in Korean. In ed. D. Baumer, D. Montero, and M. Scanlon, 1-14. Proceedings of the 25th West Coast Conference on Formal Linguistics (WCCFL-25), Somerville, Mass.: Cascadilla Proceedings Project.

Levelt, Willem. 1974. Formal grammars in linguistics and psycholinguistics, Janua linguarum. Series minor. Vol. 192. The Hague: Mouton, Recently reprinted by John Benjamins, isbn 978902723251 9.

Levy, Roger. 2008. Expectation-based syntactic comprehension. Cognition 106:1126-1177.

Levy, Roger, and Galen Andrew. 2006. Tregex and Tsurgeon: tools for querying and manipulating tree data structures. In Proceedings of the 5th International Conference on Language Resources and Evaluation (LREC 06).

Lewis, Richard L., and Shravan Vasishth. 2005. An activation-based model of sentence processing as skilled memory retrieval. Cognitive Science 29:1-45.

Lin, Chien-Jer Charles. 2008. The processing foundation of head-final relative clauses. Language and Linguistics 9.4:813-839.

Lin, Chien-Jer Charles. 2014. Effect of thematic order on the comprehension of Chinese relative clauses. Lingua 140:180-206.

Lin, Chien-Jer Charles. (submitted). Subject prominence and processing dependencies in pronominal relative clauses: The comprehension of possessive relative clauses and adjunct relative clauses in Mandarin Chinese.

Lin, Chien-Jer Charles, and Thomas Bever. 2006. Subject preference in the processing of relative clauses in Chinese. In Proceedings of the $25^{\text {th }}$ WCCFL, 254-260.

Lin, Chien-Jer Charles, and Thomas Bever 2007. Processing doubly-embedded head-final relative clauses. In Interdisciplinary Approaches to Relative Clauses, Cambridge, UK.

Lin, Chien-Jer Charles, and Thomas Bever. 2011. Garden path and the comprehension of head-final relative clauses. In Processing and Producing Head-final Structures, Studies in Theoretical Psycholinguistics, ed. H. Yamashita, Y. Hirose, and J. L. Packard, 277-297. Springer.

Lin, Yowyu Brian, and Susan Garnsey 2011. Animacy and the resolution of temporary ambiguity in relative clause comprehension in Mandarin. In Processing and Producing Head-final Structures, Studies in Theoretical Psycholinguistics, ed. H. Yamashita, Y. Hirose, and J. L. Packard, 241-276. Springer.

MacDonald, Maryellen, and Morten Christiansen. 2002. Reassessing working memory: A reply to Just and Carpenter and Waters and Caplan. Psychological Review 109(1):35-54.

MacWhinney, Brian. 1977. Starting points. Language 53:152-168.

MacWhinney, Brian. 1982. Basic syntactic processes. In Language Acquisition, Syntax and Semantics, ed. S. Kuczaj, Vol. 1, Hillsdale, NJ.: Lawrence Erlbaum.

Mak, Willem M., Wietske Vonk, and Herbert Schriefers. 2002. The Influence of Animacy on Relative Clause Processing. Journal of Memory and Language 47(1):50-68.

Mecklinger, Axel, Herbert Schriefers, Karsten Steinhauer, and Angela Friederici. 1995. Processing relative clauses varying on syntactic and semantic dimensions: An analysis with event-related potentials. Memory and Cognition 23(4):477-94.

Michaelis, Jens. 2001. On formal properties of Minimalist Grammars. Ph. D. thesis, University of Potsdam, Potsdam, Germany.

Mitchell, Don, Fernando Cuetos, Martin Corley, and Marc Brysbaert. 1995. Exposure-based models of human parsing: Evidence for the use of coarse-grained (nonlexical) statistical records. Journal of Psycholinguistic Research 24:469-488.

Miyamoto, Edson, and Michiko Nakamura. 2003. Subject/object asymmetries in the processing of relative clauses in Japanese. In The 22nd West Coast Conference on Formal Linguistics (WCCFL-22), University of California, San Diego, 342-355.

Miyamoto, Edson, and Michiko Nakamura. 2013. Unmet Expectations in the Comprehension of Relative Clauses in Japanese. In Proceedings of the 35th Annual Meeting of the Cognitive Science Society. 
Nederhof, Mark-Jan and Giorgio Satta. 2008. Computing partition functions of PCFGs. Research on Language and Computation 6:139-162.

Ning, Chunyan. 1993. The Overt Syntax of Relativization and Topicalization in Chinese. Ph. D. Dissertation, University of California, Irvine.

O'Grady, William. 1997. Syntactic Development. The University of Chicago Press.

Packard, Jerome L., Zheng Ye, and Xiaolin Zhou. 2011. Filler-gap processing in Mandarin relative clauses: Evidence from event-related potentials. In Processing and Producing Head-final Structures, Studies in Theoretical Psycholinguistics, ed. H. Yamashita, Y. Hirose, and J. Packard, Vol 38, 219-240. Springer.

Qiao, Xiaomei, Liyao Shen, and Kenneth Forster. 2012. Relative clause processing in Mandarin: Evidence from the maze task. Language and Cognitive Processes 27(4):611-630.

Schachter, Paul. 1973. Focus and relativization. Language 49:19-46.

Schriefers, Herbert, Angela Friederici, and Katja Kühn. 1995. The Processing of Locally Ambiguous Relative Clauses in German. Journal of Memory and Language 34:499-520.

Sheldon, Amy. 1974. On the role of parallel function in the acquisition of relative clauses in English. Journal of Verbal Learning and Verbal Behavior 13:272-281.

Smith, Raoul N. 1973. Probabilistic Performance Models of Language. Mouton.

Stabler, Edward. 1997. Derivational minimalism. In Logical Aspects of Computational Linguistics, ed. C. Retoré, Springer-Verlag.

Stromswold, Karin, David Caplan, Nathaniel Alpert, and Scott Rauch. 1996. Localization of syntactic comprehension by positron emission tomography. Brain and Language 52:452-473.

Traxler, Matthew, Robin Morris, and Rachel Seely. 2002. Processing subject and object relative clauses: Evidence from eye movements. Journal of Memory and Language 47:69-90.

Ueno, Mieko and Susan Garnsey. 2008. An ERP study of the processing of subject and object relative clauses in Japanese. Language and Cognitive Processes 23(5):646-688.

Vasishth, Shravan, Zhong Chen, Qiang Li, and Guilan Guo. 2013. Processing Chinese Relative Clauses: Evidence for the Subject-Relative Advantage. PLoS ONE 8(10):e77006.

Vergnaud, Jean-Roger. 1974. French Relative Clauses. Ph. D. Dissertation, Massachusetts Institute of Technology.

Wanner, E. and M. Maratsos. 1978. An ATN approach in comprehension. In Linguistic theory and psychological reality, 119-161. Cambridge Mass.: MIT Press.

Whitman, John. 2012. The prenominal relative clause problem. In Workshop on Formal Altaic Linguistics (WAFL) 8, MIT Working Papers in Linguistics (MITWPL), ed. U. Özge. Cambridge, Mass.

Wilson, Kellogg and John B. Carroll. 1954. Applications of entropy measures to problems of sequential structure. In Psycholinguistics: a survey of theory and research, ed. C. E. Osgood and T. A. Sebeok, 103-110, Indiana University Press.

Wu, Fuyun. 2009. Factors Affecting Relative Clause Processing in Mandarin: Corpus and Behavioral Evidence. Ph. D. Dissertation, University of Southern California.

$\mathrm{Wu}$, Fuyun and Elsi Kaiser (Submitted). Effects of early cues on the processing of Chinese relative clauses: Evidence for experience-based theories.

Wu, Fuyun, Elsi Kaiser, and Elaine Andersen. 2012. Animacy effects in Chinese relative clause processing. Language and Cognitive Processes 27(10):1489-1524.

Wu, Xiu-Zhi Zoe. 2000. Grammaticalization and the Development of Functional Categories in Mandarin. $\mathrm{Ph}$. D. Dissertation, University of Southern California.

Xue, Nianwen, Fei Xia, Fu-Dong Chiou, and Martha Palmer. 2005. The Penn Chinese TreeBank: Phrase structure annotation of a large corpus. Natural Language Engineering 11(2):207-238, doi:10.1017/ S135132490400364X.

Yun, Jiwon, John Whitman, and John Hale. 2010. Subject-object asymmetries in Korean sentence comprehension. In Proceedings of the 32nd Annual Meeting of the Cognitive Science Society. ed. S. Ohlsson and R. Catrambone. 